\title{
Microstructured and Photonic Bandgap Fibers for Applications in the Resonant Bio- and Chemical Sensors
}

\author{
Maksim Skorobogatiy \\ Département de Génie Physique, Ecole Polytechnique de Montreal, C. P. 6079, succ. Centre-ville, Montréal, QC, Canada H3C 3A7 \\ Correspondence should be addressed to Maksim Skorobogatiy, maksim.skorobogatiy@polymtl.ca
}

Received 3 March 2009; Accepted 2 June 2009

Recommended by Christos Riziotis

\begin{abstract}
We review application of microstructured and photonic bandgap fibers for designing resonant optical sensors of changes in the value of analyte refractive index. This research subject has recently invoked much attention due to development of novel fiber types, as well as due to development of techniques for the activation of fiber microstructure with functional materials. Particularly, we consider two sensors types. The first sensor type employs hollow core photonic bandgap fibers where core guided mode is confined in the analyte filled core through resonant effect in the surrounding periodic reflector. The second sensor type employs metalized microstructured or photonic bandgap waveguides and fibers, where core guided mode is phase matched with a plasmon propagating at the fiber/analyte interface. In resonant sensors one typically employs fibers with strongly nonuniform spectral transmission characteristics that are sensitive to changes in the real part of the analyte refractive index. Moreover, if narrow absorption lines are present in the analyte transmission spectrum, due to Kramers-Kronig relation this will also result in strong variation in the real part of the refractive index in the vicinity of an absorption line. Therefore, resonant sensors allow detection of minute changes both in the real part of the analyte refractive index $\left(10^{-6}-10^{-4} \mathrm{RIU}\right)$, as well as in the imaginary part of the analyte refractive index in the vicinity of absorption lines. In the following we detail various resonant sensor implementations, modes of operation, as well as analysis of sensitivities for some of the common transduction mechanisms for bio- and chemical sensing applications. Sensor designs considered in this review span spectral operation regions from the visible to terahertz.
\end{abstract}

Copyright (C) 2009 Maksim Skorobogatiy. This is an open access article distributed under the Creative Commons Attribution License, which permits unrestricted use, distribution, and reproduction in any medium, provided the original work is properly cited.

\section{Introduction}

R\&D into fiber-optic bio- and chemosensors (FOSs) has made a lot of progress during the last ten years. This is due to the appealing properties of FOS such as immunity to electromagnetic interference, safety in explosive environments, and potential to provide continuous quantitative and qualitative real-time analysis. Chemically sensitive thin films deposited on selected areas of optical fibers can influence the propagation of light in such fibers depending on the presence or absence of chemical molecules in the surrounding environment [1]. A wide range of optical sensors has been developed for selective biomolecule detection. Most of them have reliability issues as they employ very fragile antibodies as sensing elements. These sensors include high refractive index waveguides [2], surface plasmon resonance sensors [3], resonant mirrors [4], and classical fiber-optical sensors $[5,6]$. Most optical sensors are based on evanescent wave sensing, where the perturbations in the refractive index close to the sensor surface are probed by the exponentially decaying optical wave. Such sensors have proven to be highly sensitive in detection of small targets such as proteins and viruses, but they experience difficulties in detecting larger targets such as bacteria $(0.5-5 \mu \mathrm{m})$ since in that case much larger penetration of the evanescent field into analyte is required [7].

Microstructured Optical Fibers (MOFs), and Photonic Bandgap (PBG) fibers which are a subset of MOFs, promise a viable technology for the mass production of highly integrated and intelligent sensors in a single manufacturing step. In standard total internal reflection (TIR) fiber-based evanescent-wave sensors the fiber polymer jacket is stripped and the fiber cladding is polished to the core in order to obtain an overlap between the optical field and analyte, 
with sensor sensitivity proportional to such an overlap. Compared to the conventional solid core optical fibers, MOFs offer a number of unique advantages in sensing applications. A defining feature of a microstructured fiber is the presence of air holes running along its entire length. Fiber optical properties are then determined by the size, shape, and relative position of the holes. Particularly, a unique ability of MOFs is to accommodate biological and chemical samples in gaseous or liquid forms inside of the air holes in the immediate vicinity of the fiber core [8-10]. In this context a MOF is used simultaneously as a light guide and as a fluidic channel. The MOF's unique architecture makes it a very promising sensing platform for chemical and biological detection. First, MOFs naturally integrate optical detection with the mirofluidics, allowing for continuous online monitoring of dangerous samples in real-time without exposing the personnel to danger. In addition, the samples can be transferred in the enclosed MOF optofluidic system for further confirmation analysis, for example, polymerase chain reactions (PCRs) if needed. Such channels can be further functionalized with biorecognition layers that can bind and progressively accumulate target biomolecules, thus enhancing sensor sensitivity and specificity. Second, the MOF hole size is in sub- $100 \mu \mathrm{m}$ rage, leading to very small fluid samples required for sensing. Third, MOFbased sensors can be coiled into long sensing cells $(\sim 10 \mathrm{~m})$, thus dramatically increasing their sensitivity. The same is impossible to achieve with traditional TIR fiber sensors as side polishing step limits sensor length to several $\mathrm{cm}$. Forth, the desired MOFs can be mass-produced using commercial fiber draw tower in a very cost-effective manner. Fifth, the MOFs can potentially be scaled up into a two-dimensional array with small dimensions, which is suitable for making into portable point-of-care devices for simultaneous onsite detection of different kinds of analytes. Sixth, Photonic Bandgap fibers can be designed to guide light directly in their analyte-field hollow cores [11]. In such fibers light-analyte coupling is considerably stronger than that in evanescent sensors.

In this paper we review several MOF and PBG fiberbased resonant optical sensors, which have recently invoked strong interest due to development of novel fiber types, as well as due to development of techniques for activation of the fiber microstructure with functional materials. In resonant sensors one typically employs fibers with strongly nonuniform spectral transmission characteristics that are sensitive to changes in the real part of the analyte refractive index. Moreover, if narrow absorption lines are present in the analyte transmission spectrum, due to KramersKronig relation this will also result in strong variation in the real part of the refractive index in the vicinity of an absorption line. Therefore, resonant sensors allow detection of minute changes both in the real part of the analyte refractive index, as well as in the imaginary part of the analyte refractive index in the vicinity of absorption lines. Although the operational principle of almost all fiber-based resonant sensors relies on strong sensitivity of the fiber transmission losses to the value of the analyte refractive index, particular transduction mechanism for biodetection can vary. Consider, for example, the case of a hollow core PBG fiber featuring an analyte filled core. In one sensor implementation one can label the target biomolecules with highly absorbing particles of known absorption spectra, such as metal nanoparticles or quantum dots. The presence of such particles in the aqueous fiber core can then be quantified by detecting appearance of the absorption lines in the fiber transmission spectrum, or through resonant changes in the fiber transmission losses induced by variations in the real part of the core refractive index. In another implementation, a functional layer that binds specific biomolecules can be deposited on the inside of the hollow fiber core. Biomolecule binding events to such a layer can then be detected through resonant changes in the fiber transmission losses induced by variations in the real part of the layer refractive index.

In what follows we discuss two types of resonant sensors. One such sensor type relies on changes in the radiation losses of a leaky core mode due to changes in the real part of an analyte refractive index. Such a leaky mode is typically confined inside an analyte filled fiber core by a resonant reflector cladding. The term "leaky mode" generally refers to the guidance mechanism where the effective refractive index of a propagating mode is smaller than that of the fiber cladding. Such unusual modes are called leaky modes as, outside of a waveguide core, they do not exhibit a traditional evanescent decay into the cladding, but rather they radiate slowly (leak) into the cladding. Unlike in the case of common TIR fibers, leaky modes in PBG fibers are confined by the bandgap of a microstructured reflector. For a particular value of an analyte refractive index geometry of such a fiber is chosen to provide strong optical confinement of the leaky core mode. An example of a resonant sensor described above is a photonic bandgap fiber featuring a hollow core filled with analyte. When changing the real part of an analyte refractive index, resonant condition for mode confinement will change, resulting in strong changes in the modal radiation loss (see Figure 1(a)). Detection of changes in the transmitted intensities can be then reinterpreted in terms of the changes in the real part of an analyte refractive index. Interestingly, the same sensor can also be used in a standard nonresonant interrogation mode for the detection of changes in the imaginary part of the analyte refractive index (analyte absorption), see Figure 1(b). Even when operated in a nonresonant regime, sensitivity of the hollow core PBG fiber-based sensors is, generally, superior to that of traditional TIR fiber-based sensors due to greatly improved modal overlap with analyte.

Second sensor type considered in this review is operated in the vicinity of a phase matching wavelength between a Gaussian-like core-guided-mode and some other (highorder) mode that shows high sensitivity of its propagation properties to changes in the real part of the analyte refractive index Figure 1(b). For example, by activating fiber surface with a thin metal layer, at a specific resonant wavelength one can induce strong optical loss of a core-guided-mode due to coupling to an absorbing plasmon mode propagating at the metal/analyte interface. As plasmon mode is largely delocalized in the analyte region, wavelength of phase 

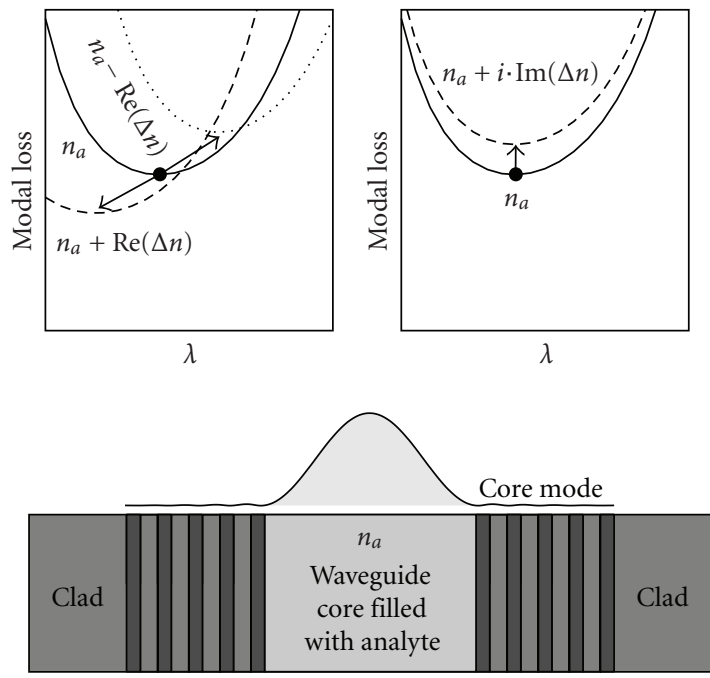

(a)
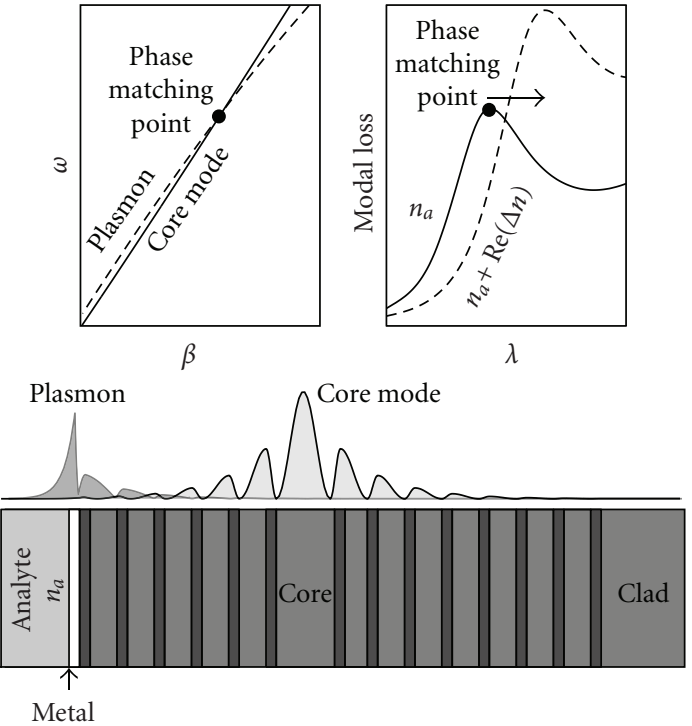

(b)

FIgURE 1: Operational principles and schematics of the two types of the resonant optical sensors. (a) Analyte-filled hollow photonic bandgap fiber-based sensor. Transmission loss through such a sensor is very sensitive to the values of both the real and imaginary parts of the analyte refractive index. (b) Sensor operating near the phase matching point of a core-guided-mode and a second mode featuring large overlap with the analyte region. In the case of a phase matching with a plasmon mode, propagation loss of a core-guided-mode is strongly dependent on the real part of the analyte refractive index.

matching between the two modes will be very sensitive to the value of the real part of analyte refractive index.

\section{Detection Strategies for Absorption-Based Sensors}

We now remind the reader some general facts about amplitude-based and spectral-based detection methodologies. Particularly, we focus on fiber-based sensors that rely on detection of changes in the transmitted light intensity in the presence of a target analyte.

In the amplitude-based detection methodology one operates at a fixed wavelength $\lambda$ and records changes in the amplitude of a signal, which are then reinterpreted in terms of changes in the analyte refractive index. To characterize sensitivity of a fiber-based sensor of length $L$, one employs an amplitude sensitivity function $S_{a}(\lambda, L)$, which is defined as a relative change in the intensity $P(\delta, \lambda, L)$ of a transmitted light for small changes in the measurand $\delta$. Note that $\delta$ can be any parameter that influences transmission properties of a fiber sensor. This includes concentration of absorbing particles in the analyte, thickness of a biolayer that can change due to capture of specific biomolecules, as well as real or imaginary parts of the analyte refractive index. Amplitude sensitivity is then defined as

$$
S_{a}(\lambda, L)=\lim _{\delta \rightarrow 0} \frac{P(\delta, \lambda, L)-P(0, \lambda, L)}{\delta \cdot P(0, \lambda, L)}=\frac{\partial P(\delta, \lambda, L) /\left.\partial \delta\right|_{\delta=0}}{P(0, \lambda, L)} .
$$

Denoting, $\alpha(\delta, \lambda)$ to be the fiber propagation loss at a fixed value $\delta$ of a measurand, light intensity at the fiber output can be written as:

$$
P(\delta, \lambda, L)=P_{\text {in }}(\lambda) \exp (-\alpha(\delta, \lambda) L),
$$

where $P_{\text {in }}(\lambda)$ is the light intensity launched into a fiber. Substituting (2) into (1), amplitude sensitivity function can be then expressed as:

$$
S_{a}(\lambda, L)=\frac{\partial \alpha(\delta, \lambda)}{\left.\partial \delta\right|_{\delta=0} \cdot L}-
$$

As follows from (3), sensor sensitivity is proportional to the sensor length $L$. In turn, as follows from (2), the maximal sensor length is limited by the absorption loss of a fiber. Defining $P_{\text {det }}(\lambda)$ to be the power detection limit at which changes in the light intensity can still be detected reliably, the maximal sensor length allowed by the power detection limit can be calculated from (2) as

$$
L=\frac{\log \left(P_{\text {in }}(\lambda) / P_{\text {det }}(\lambda)\right)}{\alpha(0, \lambda)} .
$$

Defining $\eta_{\text {det }}(\lambda)=\log \left(P_{\text {in }}(\lambda) / P_{\text {det }}(\lambda)\right)$, maximal sensitivity allowed by the power detection limit can be written using (3) as

$$
S_{a}(\lambda)=-\eta_{\operatorname{det}}(\lambda) \frac{\partial \alpha(\delta, \lambda) /\left.\partial \delta\right|_{\delta=0}}{\alpha(0, \lambda)}
$$

In all the simulations that follow we assume that $\eta_{\text {det }}(\lambda)=1$, which allows us to characterize an inherent sensitivity of a sensor system, while separating it from the issue of a power 
budget that might bring additional sensitivity enhancement. Finally, given sensor amplitude sensitivity, to estimate sensor resolution of a measurand $\delta$, one can use expression (1). Assuming that the minimal detectable relative change in the signal amplitude is $(\triangle P / P)_{\min }$ (which is typically on the order of $1 \%$ if no advanced electronics is used), then the minimum value of a measurand that can be detected by such a sensor is

$$
\delta_{\min }=\frac{(\Delta P / P)_{\min }}{S_{a}(\lambda)}
$$

Another popular sensing methodology is spectral-based. It uses detection of displacements of spectral singularities in the presence of a measurand with respect to their positions for a zero level of a measurand. This sensing approach is particularly effective in the resonant sensor configurations that feature sharp transmission or absorption peaks in their spectra. Defining $\lambda_{p}(\delta)$ to be the position of a peak in a sensor transmission spectrum as a function of a measurand value $\delta$, spectral sensitivity function can be defined as

$$
S_{\lambda}=\frac{\partial \lambda(\delta)}{\left.\partial \delta\right|_{\delta=0}} .
$$

Given sensor spectral sensitivity, to estimate sensor resolution of a measurand $\delta$, one can use expression (7). Thus, assuming that the minimal detectable spectral shift in the peak position is $\left(\Delta \lambda_{p}\right)_{\text {min }}$ (which is typically on the order of $0.1 \mathrm{~nm}$ in the visible spectral range if no advanced optical detection is used), then the minimum value of a measurand that can be detected by such a sensor is

$$
\delta_{\min }=\frac{\left(\Delta \lambda_{p}\right)_{\min }}{S_{\lambda}} .
$$

\section{Sensing Using Analyte-Filled Hollow Core Photonic Bandgap Fibers}

We now describe the first resonant sensor type based on hollow core photonic bandgap fibers filled with analyte. In their crossection PBG fibers can contain periodic sequence of micron-sized layers of different materials [11, 12] (Figure 2(a)), periodically arranged micron-sized air voids [13-15] (Figure 2(b)), or rings of holes separated by nanosupports $[16,17]$ (Figure 2(c)). PBG fibers are currently available in silica glass, polymer and specialty soft glass implementations. The key functionality of such fibers is their ability to guide light directly in the hollow or liquid-filled cores with refractive index smaller than the refractive index of a surrounding cladding material. Unlike microstructured fibers, PBG fibers confine light in their hollow cores by photonic bandgap effect, rather than by total internal reflection. Practically, bandgaps are defined as frequency regions of enhanced fiber transmission, and they are the result of destructive interference of the coreguided light inside of the fiber-microstructured cladding. When launching spectrally broad light into a PBG fiber, only the spectral components guided by the fiber bandgaps will reach the fiber end, while all the spectral components not located within the bandgaps will be irradiated out near the fiber coupling end. Moreover, even in the absence of fiber material losses, core-guided-modes always exhibit radiation loss. This is a direct consequence of guidance in a core with refractive index smaller than that of a cladding. As we will soon see, core mode radiation loss can be very sensitive to the value of the real part of the refractive index of the material filling the fiber core, which can be utilized for sensor applications. Finally, PBG fibers have a tendency to improve the beam quality of guided light, while being effectively single mode in the limit of long propagation distances. This is a consequence of the fact that radiation losses (and, generally, absorption losses too) of the core-guided-modes of a PBG fiber are strongly differentiated with only a few low-order modes having small propagation losses. Thus, when exciting several modes at the fiber input end, only the modes having the lowest losses will survive till the fiber end. For historical reference we mention that before the invention of the alldielectric PBG fibers, guidance in the hollow core fibers has been demonstrated in the context of metal coated capillaries $[18,19]$.

We now detail some of the advantages offered by the hollow core PBG fibers for sensing applications. One has to distinguish two modes of operation of such sensors. First, is sensing of changes in the imaginary part of the analyte refractive index (analyte absorption) by detecting the presence and strength of the narrow absorption bands in the fiber transmission spectrum. This is the simplest, nonresonant application of the hollow PBG fibers for optical sensing in which one only takes advantage of large overlap of the core-guided leaky mode with analyte. In such sensors, signal strength due to analyte absorption, as well as sensor sensitivity are directly proportional to the sensor length. Recently, several experimental implementations of such absorption-based sensors have been demonstrated [10, 2023]. Second mode of operation of a PBG fiber-based sensor is sensing of changes in the real part of the analyte refractive index by detection of shifts in the fiber bandgap position. As it will be explained in the following, such a sensor operates in the resonant regime with sensitivity that is largely independent of the sensor length.

3.1. Nonresonant Sensing. Classic perturbation theory considerations [24] predicts that changes in the effective refractive index of a guided mode $\Delta n_{\text {eff }}$ are related to the changes in the refractive index $\Delta n_{a}$ of analyte infiltrating the fiber, through the overlap factor $f$ defined as

$$
\begin{gathered}
\Delta n_{\mathrm{eff}}=\Delta n_{a} \cdot f=\operatorname{Re}\left(\Delta n_{a}\right) \cdot f+i \cdot \operatorname{Im}\left(\Delta n_{a}\right) \cdot f \\
f=\frac{\int_{\text {analyte }} d A|\mathbf{E}|^{2}}{\operatorname{Re}\left(\hat{\mathbf{z}} \cdot \int_{\text {crossection }} d A \mathbf{E}_{t}^{*} \times \mathbf{H}_{t}\right)},
\end{gathered}
$$

where $\mathbf{E}_{t}, \mathbf{H}_{t}$ are the transverse electromagnetic fields of a fiber mode, while $\mathbf{E}$ is a complete electric field of a mode. Strictly speaking, expression (9) is only valid for the truly guided square integrable modes of the Total Internal Reflection (TIR) fibers. In the case of hollow-core PBG fibers 


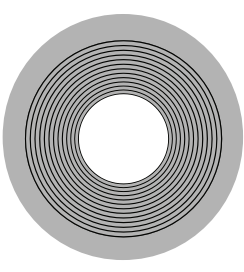

(a)

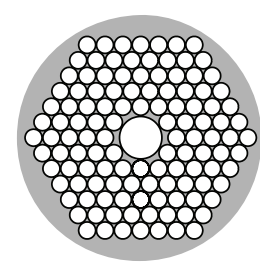

(b)

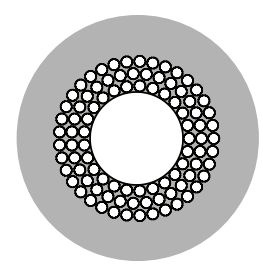

(c)

Figure 2: Various types of hollow core photonic bandgap fibers. (a) Bragg fiber featuring large hollow core surrounded by a periodic sequence of high and low refractive index layers. (b) Photonic crystal fiber featuring small hollow core surrounded by a periodic array of large air holes. (c) Microstructured fiber featuring medium size hollow core surrounded by several rings of small air holes separated by nano-size bridges.

the leaky modes are, generally, nonsquare integrable [25]. In this case, however, expression (9) can still be used but only approximatively. Particularly, to avoid divergence in the denominator of (9), one performs integration only over the finite fiber crossection limited by the interface between the multilayer reflector and a cladding. For the hollow core PBG fibers, detailed simulations show that $f$ is typically larger than 0.9. The value of an overlap increases rapidly when the fiber core size increases, reaching values higher than 0.99 for even the moderate core sizes $R_{\text {core }} \sim 5-10 \lambda$. Such a high value of the overlap factor is explained by high confinement of the guided mode in the fiber core (see, e.g., energy flux distribution of the core-guided-mode in the inset of Figure 1(a)).

Expression (9) is fundamental for the analysis of nonresonant absorption-based sensors. Consider, for example, a microstructured or a hollow core fiber filled with aqueous solution. One possible biosensor implementation utilizing such fibers can, for example, monitor presence and concentration of specific biomolecules labeled by highly absorbing nanoparticles. In such a sensor biomolecules in the aqueous solution are purged through the fiber microstructure. Defining $C$ to be the concentration (measurand; $\delta=C$ in (1)) of the absorbing particles mixed with analyte, and assuming that nano-particle bulk absorption per unit of concentration is $\alpha_{C}(\lambda)$, while fiber loss in the absence of nanoparticles is $\alpha_{f}(\lambda)$, then total fiber loss in the presence of absorbing nanoparticles can be written using (9) as

$$
\alpha(C, \lambda)=\alpha_{f}(\lambda)+f \cdot C \cdot \alpha_{C}(\lambda) .
$$

In derivation of (10) we used the fact that $\operatorname{Im}\left(\Delta n_{a}\right) \sim C \cdot \alpha_{C}$, $\alpha(C, \lambda) \sim \operatorname{Im}\left(n_{\text {eff }}\right)$. By substituting (10) into (5), we now find expression for the maximal nonresonant sensor sensitivity to changes in the nano-particle concentration:

$$
S_{a}(\lambda)=-f \frac{\alpha_{C}(\lambda)}{\alpha_{f}(\lambda)}
$$

Note that as nano-particle absorption $\alpha_{C}(\lambda)$ is completely independent from the fiber loss $\alpha_{f}(\lambda)$ in the absence of nanoparticles, sensitivity (11) of a nonresonant sensor is, thus, directly proportional to the fiber length $L \sim 1 / \alpha_{f}(\lambda)$. Consequently, to increase sensor sensitivity one has to simply work with longer fibers featuring low propagation loss.
3.2. Resonant Sensing. Note that expression (9), when applied to PBG fibers, does not account for the spectral shift of the PBG fiber bandgap (see Figure 1(a)) due to changes in the real part of the refractive index of an analyte filling the fiber core. In fact, for the hollow-core PBG fibers in place of (9) one has to use the following modified expression:

$$
\Delta n_{\text {eff }}=\operatorname{Re}\left(\Delta n_{a}\right) \cdot f+i\left[\operatorname{Im}\left(\Delta n_{a}\right) \cdot f+\operatorname{Re}\left(\Delta n_{a}\right) \cdot f_{\mathrm{rad}}\right] .
$$

Here, $f_{\text {rad }}$ is a radiation factor that describes changes in the radiation losses of a photonic bandgap guided mode due to spectral shift of a fiber bandgap caused by changes in the real part of the refractive index of an analyte filling the fiber core.

To understand the radiation loss contribution in (12) one has to recall the principles of design and operation of the hollow-core PBG fibers. Consider, as an example, the case of a hollow core plastic Bragg fiber featuring a water filled core (refractive index $n_{w}$ ) surrounded by a Bragg reflector (Figure 3(a)) made of a periodic sequence of two optically different materials with refractive indices $n_{l}, n_{h}$ which are assumed to be purely real $[11,26]$. We now design Bragg reflector to feature the fundamental bandgap in the visible at $\lambda_{0}=0.5 \mu \mathrm{m}$. The reflector layer thicknesses $d_{l}, d_{h}$ have to be chosen in a very specific way as to guarantee the destructive interference of guided light in the periodic fiber cladding, hence efficient modal confinement in the fiber hollow core. Particularly, by choosing the reflector layer thicknesses to satisfy the quarter wave condition:

$$
d_{l, h}=\frac{\lambda_{0}}{4 \sqrt{n_{l, h}^{2}-\operatorname{Re}\left(n_{\text {core }}\right)^{2}}},
$$

one guarantees that the fundamental bandgap of a Bragg reflector is centered in the near vicinity of a design wavelength $\lambda_{0}$ [25].

We now consider particular implementation of a Bragg fiber having the core of radius $R_{\text {core }}=25 \mu \mathrm{m}$ surrounded by 6 reflector layers with $n_{h}=1.6, n_{l}=1.4$ and the layer thicknesses given by (13), where $n_{\text {core }}=1.34$. In Figure 2(b) in thick solid curve we present propagation loss of the fundamental Gaussian-like $H E_{11}$ core mode of a Bragg fiber. In fact, $H E_{11}$ mode plays a key role in the operation of a majority of the hollow-core based sensors as it is the easiest mode to excite with an external Gaussian-like laser source. 


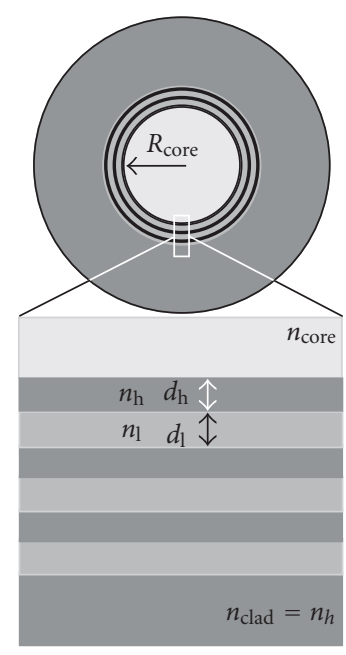

(a)

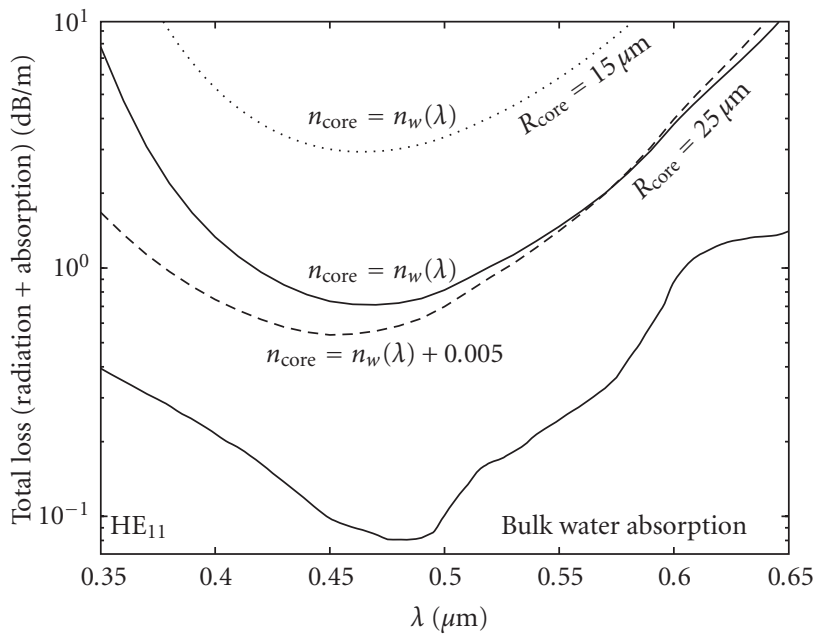

(b)

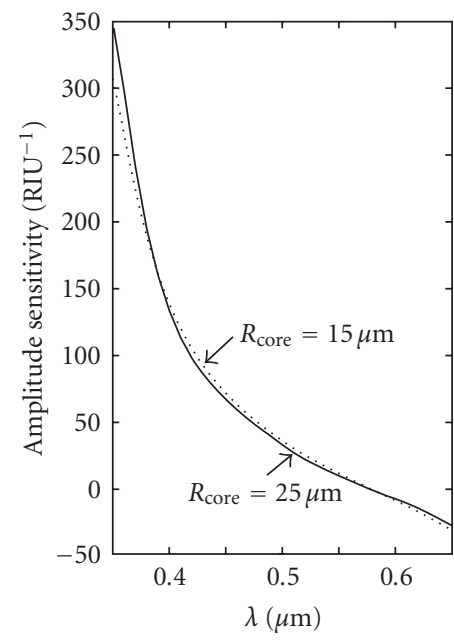

(c)

Figure 3: Refractive index sensors based on hollow core PBG Bragg fibers. (a) Schematic of a hollow core Bragg fiber filled with aqueous analyte of refractive index $n_{w}(\lambda)$. (b) Radiation-dominated propagation loss of a core-guided $H E_{11}$ mode for two Bragg fibers with core radii $R_{\text {core }}=25 \mu \mathrm{m}$ (solid curve) and $R_{\text {core }}=15 \mu \mathrm{m}$ (thin dashed curve). When analyte refractive index changes, so does the radiation loss of a fiber core mode (thick dashed curve). For the reference, in thin solid line we present absorption loss of distilled water in the visible. (c) Maximal amplitude sensitivity of the analyte filled Bragg fiber-based sensor for the two values of the fiber core radius. Note that amplitude sensitivity is almost independent of the fiber core radius.

While total propagation loss is a sum of the modal radiation and absorption losses, this particular fiber is operating in the Radiation-dominated regime; for comparison, bulk absorption loss of water is presented in Figure 3(b) in thin solid line. We note that modal propagation loss reaches its minimum at the center of a photonic bandgap at $\sim 0.46 \mu \mathrm{m}$, while increasing rapidly towards the edges of a bandgap. When changing the real part of the refractive index of an analyte filling the fiber core, resonant condition (13) is no longer satisfied, thus leading to spectral displacement of the fiber bandgap (a thick dashed line in Figure 3(b)). Therefore, even for small changes in the analyte refractive index, due to spectral shift in the position of a reflector bandgap, fiber propagation loss can vary substantially. Moreover, due to resonant nature of bandgap guiding, bandgap shift due to changes in the real part of the analyte refractive index can have a substantially stronger effect on the propagation losses of a core-guided-mode than changes in the absorption coefficient of an analyte. Particularly, in (13) one can calculate that at the operational wavelength of $0.5 \mu \mathrm{m}, f \simeq 1$, while $f_{\text {rad }} \simeq 2.4 \cdot 10^{-4}$. Therefore, change of $1 \mathrm{~dB} / \mathrm{m}$ loss in the analyte absorption coefficient $\left(\operatorname{Im}\left(\Delta n_{a}\right) \simeq 0.9 \cdot 10^{-8}\right)$, or change of $\operatorname{Re}\left(\Delta n_{a}\right) \simeq 3.8 \cdot 10^{-5}$ in the real part of the analyte refractive index, result in the same $1 \mathrm{~dB} / \mathrm{m}$ change in the modal propagation loss.

We now investigate amplitude and spectral sensitivities of the hollow-core PBG fiber-based sensors. In Figure 3(c) we present amplitude sensitivity (5) of a Bragg fiber-based sensor to changes in the real part of the analyte refractive index $\delta=\operatorname{Re}\left(\Delta n_{a}\right)$. Note that sensitivity varies strongly as a function of the wavelength of operation, increasing rapidly towards the bandgap edges. Inside of a bandgap and in the vicinity of a design wavelength, amplitude sensitivity is on the order of $S_{a} \sim 100 \mathrm{RIU}^{-1}$. Assuming that $1 \%$ of change in the amplitude of a transmitted light can be detected reliably, this results in sensor resolution of $\operatorname{Re}\left(\Delta n_{a}\right)_{\min } \sim 10^{-4}$ RIU. Finally, in the vicinity of a bandgap center at $470 \mathrm{~nm}$ the total fiber loss is $\sim 0.7 \mathrm{~dB} / \mathrm{m}$, thus defining the maximal sensor length to be on the order of $\sim 6 \mathrm{~m}$.

In a similar fashion, spectral sensitivity can be defined using (7) by detecting spectral shift in the bandgap center (wavelength of the fiber lowest loss) resulting in a spectral sensitivity $S_{\lambda} \sim 5300 \mathrm{~nm} / \mathrm{RIU}$. Assuming that $0.1 \mathrm{~nm}$ spectral shift in the position of a bandgap center can be detected reliably, this results in the sensor resolution of $\operatorname{Re}\left(\Delta n_{a}\right)_{\min } \sim$ $2 \cdot 10^{-5} \mathrm{RIU}$, which is comparable to the resolution achieved by the amplitude method.

Interestingly, maximal sensitivity of a resonant hollowcore PBG fiber sensor does not depend strongly on the fiber length. To demonstrate that, in Figure 3(b) we also present losses of an $H E_{11}$ mode of a hollow core Bragg fiber, with a core radius $R_{\text {core }}=15 \mu \mathrm{m}$ and otherwise identical parameters to the Bragg fiber with $R_{\text {core }}=25 \mu \mathrm{m}$. Note that losses of a smaller core fiber are almost 10 times as high as losses of a larger core fiber. This signifies that the maximal sensor length for a smaller core fiber is almost 10 times shorter than the length of a sensor based on a larger core fiber. In Figure 3(c) we also plot the maximal sensitivity (5) of a Bragg fiber-based sensor with a core radius $R_{\text {core }}=15 \mu \mathrm{m}$ and note that it is almost identical to that of a larger core fiber. This interesting, while somewhat counterintuitive result is a direct consequence of the fact that in PBG fibers operating in the Radiation-dominated regime, fiber propagation loss and radiation factor are not independent parameters. Particularly, starting from (12), and assuming that the fiber loss for a neutral analyte is $\alpha_{f}(\lambda)$, 
then PBG fiber absorption loss $\alpha\left(\Delta n_{a}, \lambda\right)$ in the presence of changes in the real part of the analyte refractive index is described by:

$$
\alpha\left(\Delta n_{a}, \lambda\right)=\alpha_{f}(\lambda)+\frac{4 \pi}{\lambda} \operatorname{Re}\left(\Delta n_{a}\right) \cdot f_{\mathrm{rad}} .
$$

In the case of Bragg fibers operating in the Radiationdominated regime one generally finds that $f_{\text {rad }} \sim \alpha_{f}(\lambda)$. Therefore, maximal amplitude sensitivity as defined by (5) will not depend on the fiber loss, and as a consequence, it will not depend on the sensor length. This finding promises a significant advantage of PBG fiber based resonant sensors compared to their conventional absorption-based counterparts. Particularly, compact and highly sensitive sensors that utilize short PBG fiber pieces and that do not require fiber coiling are more convenient to use, and easier to maintain than their conventional counterparts that utilize long coiled fibers.

\section{Surface Plasmon Resonance-Based Fiber Sensors}

In this section we describe another type of a resonant sensor operating in the vicinity of a phase matching point between a Gaussian-like core mode of a fiber (waveguide) and another mode that has strong overlap with analyte region (see the inset of Figure 1(b)). In what follows, the mode that has strong overlap with analyte is assumed to be a plasmonic wave propagating on the metalized fiber surface facing analyte.

Propagating at the metal/dielectric interface, surface plasmons [27] are extremely sensitive to changes in the refractive index of the dielectric. This feature constitutes the core of many Surface Plasmon Resonance (SPR) sensors. Typically, these sensors are implemented in the Kretschmann-Raether prism geometry where p-polarized light is launched through a glass prism and reflected from a thin metal $(\mathrm{Au}, \mathrm{Ag})$ film deposited on the prism facet [28]. The presence of a prism allows phase matching of an incident electromagnetic wave with a plasmonic wave at the metal/ambient dielectric interface at a specific combination of the angle of incidence and wavelength. Mathematically, phase matching condition is expressed as an equality between the plasmonvector and a projection of the wavevector of an incident wave along the interface. Since plasmon excitation condition depends resonantly on the value of the refractive index of an ambient medium within 100-300 nm from the interface, the method enables, for example, detection, with unprecedented sensitivity, of biological binding events on the metal surface [29]. The course of a biological reaction can then be followed by monitoring angular [29, 30], spectral [31] or phase $[32,33]$ characteristics of the reflected light. However, the high cost and large size of commercially available systems makes them useful mostly in a laboratory, while many important field and other applications still remain out of the reach for this method.

Using optical waveguides and fibers instead of bulk prism configuration in plasmonic sensors offers miniaturization, high degree of integration and remote sensing capabilities. In fiber and waveguide-based sensors, one launches the light into a waveguide core and then uses coupling of a guided mode with a plasmonic mode to probe for the changes in the ambient environment. To excite efficiently a surface plasmon, the phase matching condition between a plasmon and a waveguide mode has to be satisfied, which mathematically amounts to the equality between their modal propagation constants (effective refractive indices). Over the past decade, driven by the need for miniaturization of SPR sensors, various compact configurations enabling coupling between optical waveguide modes and surface plasmonic waves have been investigated. Among others, metalized single mode, multimode and polarization maintaining waveguides and fibers, metalized tapered fibers, metalized fiber Bragg gratings [34-49] and, recently, solid core microstructured fibers [50-52], as well as planar photonic crystal waveguides [53] have been studied. In the majority of fiber implementations (with an exception of microstructured fibers), one typically strips fiber polymer jacket and polishes off fiber cladding until fiber core is exposed; then, a metal layer is deposited directly onto a fiber core. Thus functionalized surface of a fiber core is then exposed to an analyte.

Ideally, one would use a single mode fiber or waveguide with all the power traveling in a single Gaussian-like core mode operating near the point of resonant excitation of the plasmon [37, 54-58]. Gaussian shape of a core mode is important as it is best suited for the excitation by standard Gaussian laser sources. Near the point of phase matching, most of the energy launched into a waveguide core mode should be efficiently transferred into a plasmon mode. However, in the Total Internal Refraction (TIR) single mode waveguides with low refractive index-contrast, coupling with a plasmon is realized at essentially grazing angles of modal incidence on the metal layer. As follows from the basic SPR theory, coupling at such grazing incidence angles leads to an inevitable decrease of sensitivity of the SPR method. In principle, high index-contrast single mode waveguides (see Figure 4(a)) could be employed to increase the angle of modal incidence on the interface. However, in the single mode waveguide-based sensors, phase matching between plasmon and fundamental waveguide mode is typically hard to realize. This is related to the fact that the effective refractive index of a core-guided-mode is close to the refractive index of the core material, which is typically larger than 1.45 due to practical material limitations. The effective refractive index of a plasmon is close to the refractive index of an ambient medium which is typically air $n_{a}=1$ (gas sensing) or water $n_{a}=1.33$ (biological sensing). Thus, large discrepancy in the effective refractive indices makes phase matching between the two modes hard to achieve, with an exception of the high frequencies $\lambda<600 \mathrm{~nm}$, where the plasmon dispersion relation starts deviating towards higher refractive indices. Thus, due to practical limitation on the lowest value of the waveguide core and cladding refractive indices, single mode TIR waveguide-based sensors were demonstrated almost exclusively in the visible where phase matching condition is easier to enforce. 


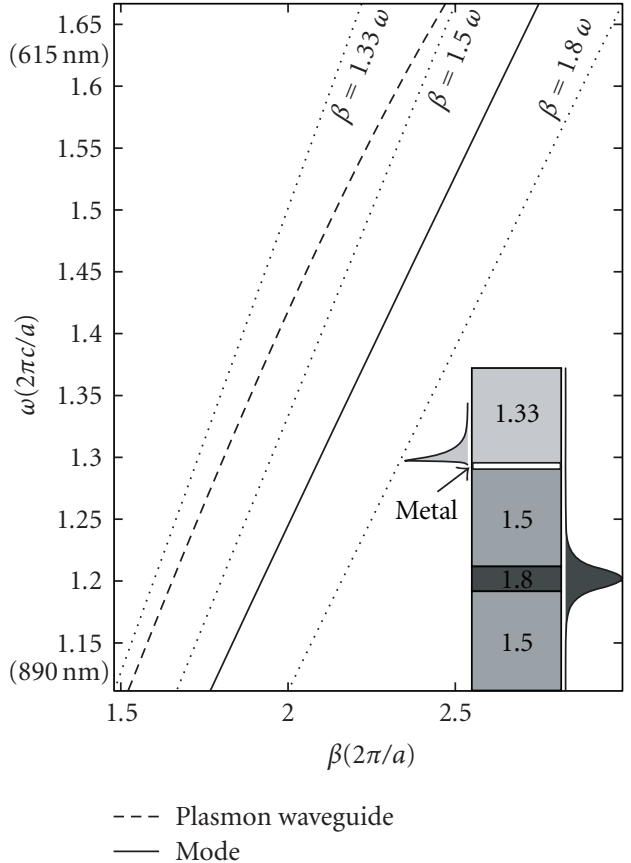

(a)

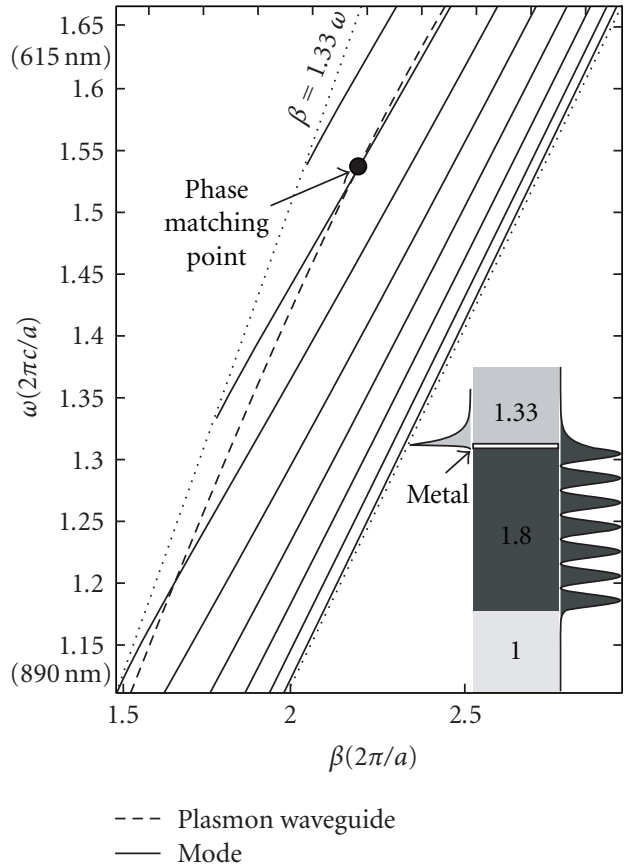

(b)

FIGURE 4: Band diagrams and schematics of various SPR-based integrated sensor implementations. (a) Single mode waveguide-based sensor. Dispersion relations of a core-guided-mode (solid) and a plasmon (thick dashed). Inset-sensor schematic; $\left|H_{\|}\right|^{2}$ of a plasmon (a) and a core mode (b). (b) Multimode waveguide-based sensor. Dispersion relations of the core modes (solid) and a plasmon (thick dashed). Insetcoupler schematic; $\left|H_{\|}\right|^{2}$ of a plasmon (a) and a high-order mode (b) at the phase matching point (black circle).

Problems with phase matching and loss of sensitivity due to shallow angles of incidence could be, in principle, alleviated by using multimode waveguides [45-49] presented in Figure 4(b). If launched properly, modal effective propagation angles in such waveguides can be much steeper, also resulting in smaller effective refractive indices. However, in multimode waveguides, a large number of higher-order modes can be phase matched with a plasmon. Thus, sensitivity and stability of such sensors depend crucially on launch conditions. Moreover, as spatial field distribution in a Gaussian-like laser source is typically not well matched with the field distributions of higher-order modes of a multimode waveguide, only a small fraction of energy can be efficiently launched into such modes, thus resulting again in decreased sensitivity.

\section{Plasmon-Assisted Sensing Using Solid Core Microstructured Fibers}

To alleviate the problem of phase matching of the fiber core mode and a plasmon one approach is to introduce additional geometrical features into the fiber core that could allow tuning of the fiber core mode effective refractive index $[51,59]$. As mentioned before, in the case of aqueous or gaseous analytes the core mode effective refractive index has to be lowered to match that of a plasmon. As a first example of a fiber where tuning by design can be effectively achieved we consider a solid core microstructured optical fiber shown in Figure 5(a). Such a MOF is comprised of a solid core surrounded by a ring of six air holes of diameter $d_{1}$ which are placed at the vertices of a hexagon with vertice-tovertice distance $\Lambda$. The ring of holes works as a low refractive index cladding enabling mode guidance in the fiber core. To lower the refractive index of a core-guided-mode a small air hole of diameter $d_{c}$ is introduced into the fiber core center. In place of a single hole, an array of smaller holes can be used similarly to [60]. Finally, two large semicircular channels of size $d_{2}$ surround the microstructured fiber core region. During sensor operation these channels are filled with analyte, and in what follows we assume that the analyte under study is distilled water with $n_{a} \simeq 1.33$. On a fiber core side the channels are covered with a thin gold film of thickness $d_{\text {gold }} \simeq 40 \mathrm{~nm}-60 \mathrm{~nm}$ to enable plasmon excitation at the gold/analyte interface. Coupling strength between the core mode and plasmon is strongly influenced by the size $d_{1}$ of the holes surrounding the fiber core, (larger hole sizes result in a weaker coupling). We assume that MOF is made of a silica glass with refractive index given by one of the standard Sellmeier-type fits $n_{\text {glass }} \simeq 1.46$ [61]. Finally, the dielectric constant of gold is approximated by the Drude model:

$$
\varepsilon_{\text {gold }}\left(\lambda_{c}\right)=\varepsilon_{\infty}-\frac{\left(\lambda / \lambda_{p}\right)^{2}}{1+i\left(\lambda / \lambda_{t}\right)},
$$

where the choice $\varepsilon_{\infty}=9.75, \lambda_{t}=13 \mu \mathrm{m}, \lambda_{p}=0.138 \mu \mathrm{m}$ presents one of the many possible fits of the experimental data. 
As a concrete example we consider MOF with the following parameters $\Lambda=2 \mu \mathrm{m}, d_{c}=0.5 \Lambda, d_{1}=0.6 \Lambda$, $d_{2}=0.8 \Lambda, d_{\text {gold }}=40 \mathrm{~nm}$.

Finite element method with PML boundaries was used to find complex propagation constants of the core-guided and plasmonic modes. A typical fiber-based plasmonic sensor operates in the vicinity of a phase matching point between the core-guided-mode and a plasmon localized at the metalized surface. Figure 5(b) we present dispersion relations of the core-guided-mode (thick solid line) and a plasmon (thick dashed line). Phase matching point is located at $640 \mathrm{~nm}$ where the difference between the modal refractive indexes is the smallest. Detailed simulations show that by changing the size of a central hole in the range $d_{c}=0.35 \Lambda-0.55 \Lambda$, or by changing diameters of the six holes in the range $d_{1}=0.6 \Lambda-0.7 \Lambda$, one can readily tune the effective refractive index of the fundamental mode by $0.01 \mathrm{RIU}$ which is equivalent to $30 \mathrm{~nm}$ spectral tuning of the position of a plasmonic peak. Energy flux distributions in the vicinity of a phase matching point (insets (I,II) in Figure 5(b) allow clear differentiation of the nature of the two modes as being the core mode (inset (I)) and plasmonic mode (inset (II)). Due to symmetry, only a quarter of the fiber crossection is presented in the insets. In the vicinity of a phase matching point the two modes become strongly mixed (inset (III) in Figure 5(c)), with losses of a core-guidedmode increasing dramatically due to the energy transfer into the lossy plasmon mode. Core mode losses calculated as $\operatorname{Im}\left(n_{\text {eff }}\right)$ are presented in Figure $5(\mathrm{~b})$ in a thin solid line. For the reference, losses in $[\mathrm{dB} / \mathrm{m}]$ are defined as $\alpha[\mathrm{dB} / \mathrm{m}]=$ $\left(40 \pi \operatorname{Im}\left(n_{\text {eff }}\right)\right) /(\ln (10) \lambda[\mathrm{m}])$.

As explained earlier, detection of changes in the propagation loss of a core-guided-mode at the point of its phase matching with a plasmon constitutes the core of many SPR sensor implementations. We now detail plasmon-based transduction mechanism in greater details. In Figure 6(a) we present losses of the fundamental core-guided-mode in the wavelength range of $0.5 \mu \mathrm{m}-1.3 \mu \mathrm{m}$ for the same MOF design as discussed earlier. Solid line corresponds to the case of a pure analyte with $n_{a} \simeq 1.33$ and it features two plasmonic excitation peaks located at $640 \mathrm{~nm}$ and $1120 \mathrm{~nm}$. To demonstrate potential of this fiber for sensing, we present in a dashed line losses of the core-guided-mode for the case when the analyte refractive index is changed to $n_{a} \simeq 1.335$. As seen from Figure 6(a) position of the first plasmonic peak (at shorter wavelengths) shifts by as much as $10 \mathrm{~nm}$, which is readily detectable. From the amount of shift in the position of a plasmonic peak one can, in principle, extract the value of changes in the analyte refractive index. This transduction mechanism is commonly used for the detection of the analyte bulk refractive index changes, as well as monitoring of formation of the nanometer-thin biolayers on top of a metalized sensor surface.

Interestingly, when analyte refractive index is varied the position of the second plasmonic peak in Figure 6(a) stays mostly unchanged. Therefore, such a peak cannot be used for sensing changes in analyte refractive index. Nevertheless, presence of such a peak is very useful as it serves as a natural reference in the measurements, which simplifies greatly data acquisition and data interpretation. To understand better the nature of the two plasmonic peaks, in the insets of Figure 6(a) we present energy flux distributions in the core-guided core modes evaluated at the first and second plasmonic peaks. From the pictures one notices that at the first peak (inset (I), Figure 6(a)) the plasmon contribution to the hybrid mode is delocalized in the analyte region, while at the second peak (inset (II), Figure 6(a)) the plasmon contribution to the hybrid mode is delocalized mostly in the fiber cladding. This explains why spectral position of the first peak is considerably more sensitive to the changes in the analyte refractive index than that of a second peak.

Finally, in Figure 6(b) we present amplitude sensitivity of the MOF sensor to changes in the real part of the analyte refractive index as defined in $(5)\left(\delta=\operatorname{Re}\left(\Delta n_{a}\right)\right)$. Sensor sensitivities are calculated for several values of the gold layer thickness. Maximal sensitivity $S_{a} \sim 180 \mathrm{RIU}^{-1}$ is achieved for a $40 \mathrm{~nm}$ thick gold film. Assuming that $1 \%$ of change in the amplitude of a transmitted light can be detected reliably, this results in sensor resolution of $\operatorname{Re}\left(\Delta n_{a}\right)_{\min } \sim$ $6 \cdot 10^{-5}$ RIU. Finally, at the wavelength of maximal sensor sensitivity at $665 \mathrm{~nm}$ the total fiber loss is $\sim 150 \mathrm{~dB} / \mathrm{cm}$, thus defining the maximal sensor length to be on the order of $\sim 1 \mathrm{~mm}$. In passing we note that plasmon being a surface excitation is very sensitive to the thickness of a metallic layer. This can be used to study metal nanoparticle binding events on the metallic surface of a sensor [62]. This mode of sensor operation can be of interest, for example, to the monitoring of concentration of metal nanoparticles attached to the photosensitive drugs in the photodynamic cancer therapy [63].

\section{Plasmon-Assisted Sensing Using Solid Core Photonic Bandgap Fibers}

As mentioned in the previous section, introduction of additional geometrical features into the fiber core could allow tuning of the fiber core mode effective refractive index. However, in the case of TIR-microstructured fibers the tuning range, while significant $(0.01 \mathrm{RIU}$ as shown in the previous section), is not sufficient to alleviate the problem of phase matching of the fiber core mode and a plasmon. The problem of phase matching becomes especially pronounced in the case of low refractive index analytes. In what follows, we detail design principles of the photonic bandgap fiber and waveguide-based SPR sensors, and show that in such sensors the fundamental Gaussian-like leaky core mode can be phase matched with a plasmon at any desired wavelength of operation from the visible to mid-IR, and for any value of the analyte refractive index. Effectively, PBG waveguidebased sensors integrate advantages of both the multimode and single mode waveguide-based SPR sensors by being able to reduce the core mode refractive index to any desired value (even 0 ), while operating with a Gaussian-like core mode.

To remind the reader, the term "leaky mode" generally refers to the guidance mechanism where the effective refractive index of a propagating mode is smaller than that of the waveguide cladding. Such unusual modes are called 


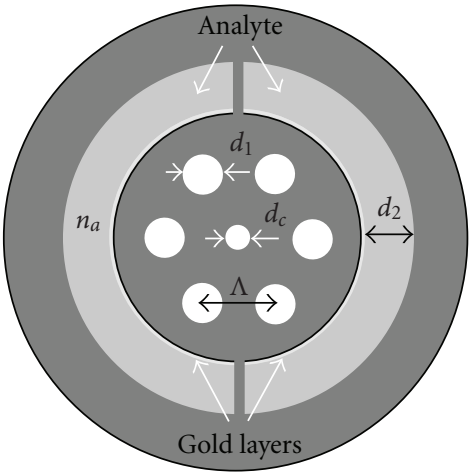

(a)

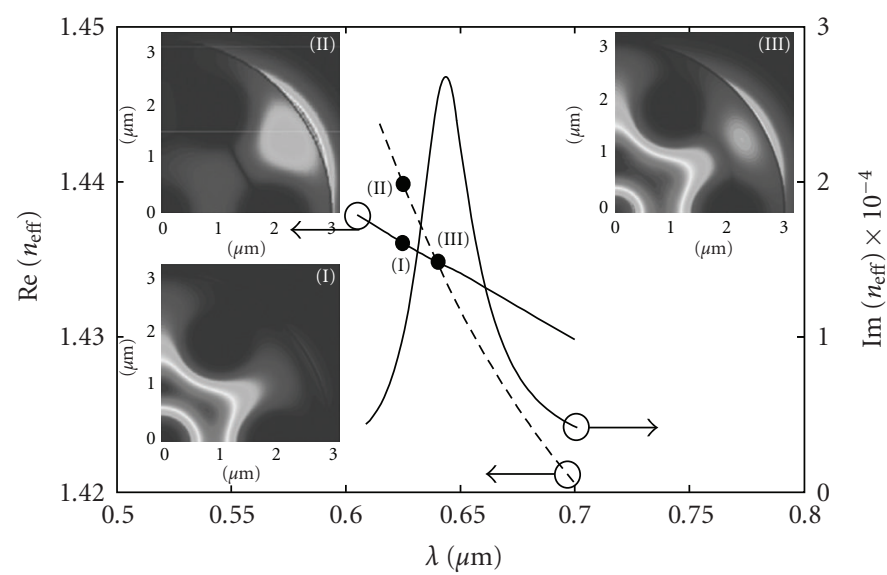

(b)

FIgURE 5: (a)Schematics of a MOF-based SPR sensor. The fiber comprises six air holes surrounding a solid fiber core. These holes form a microstructured cladding with low effective refractive index. A smaller hole is introduced in the core center to enable tunability of the core mode effective refractive index to facilitate phase matching with a plasmon. Two large semicircular metalized channels are integrated into the fiber structure to enable efficient microfluidic flow of analyte and plasmon excitation at the metal/analyte interface. (b) Dispersion relations of a core-guided-mode (thick solid line) and a surface plasmon (thick dashed line) in the vicinity of the phase matching point. Insets (I), (II) show energy fluxes of the core-guided and plasmon modes close to the phase matching point. Transmission loss of a core-guided-mode (thin solid line) exhibits strong increase at the phase matching point due to efficient mixing with a plasmon as confirmed by its energy flux distribution (inset (III)).

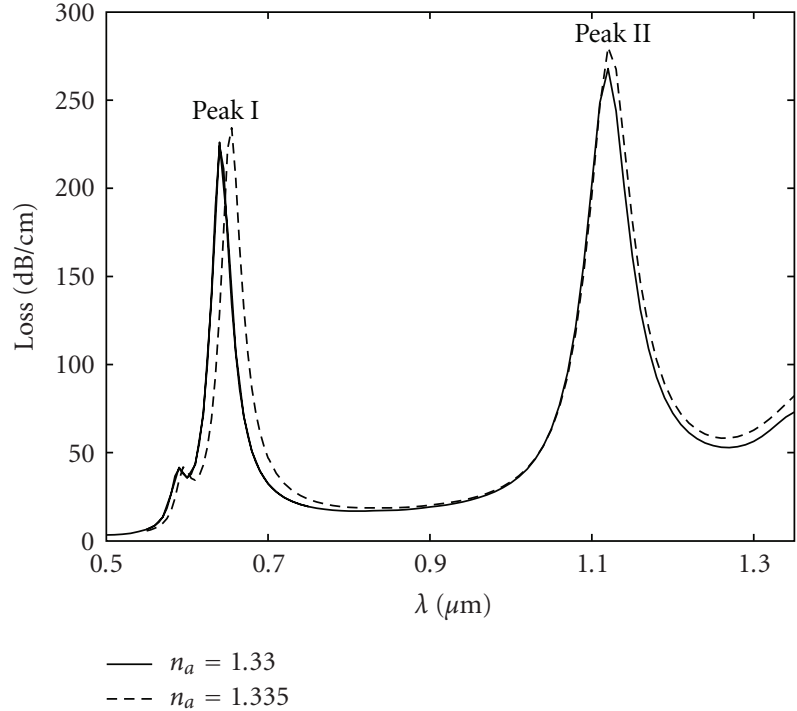

(a)
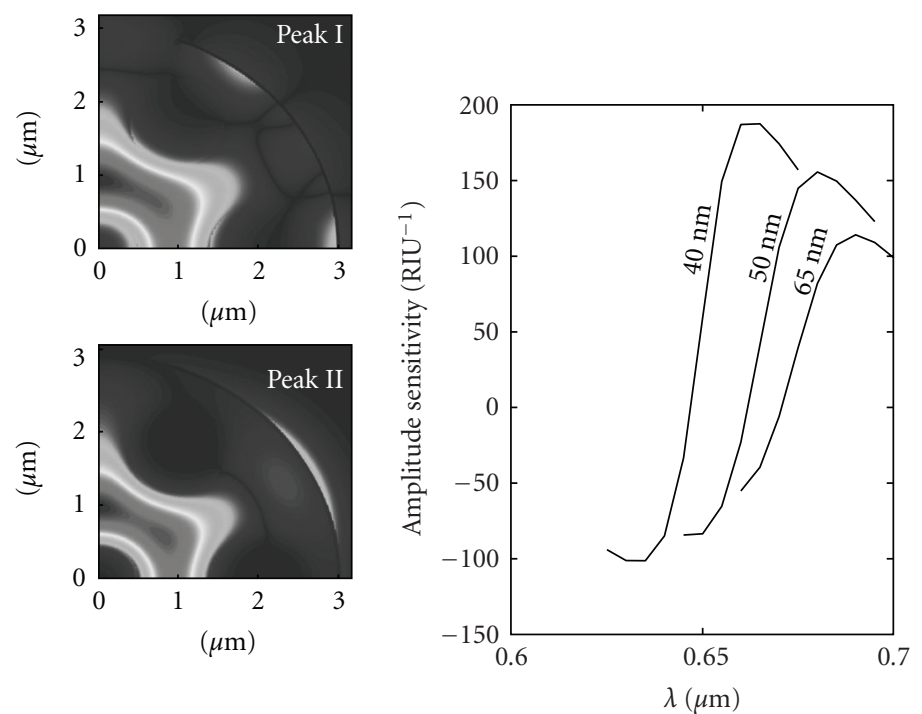

(b)

FIGURE 6: Calculated loss spectra of the fundamental core-guided-mode of a metalized MOF. Loss spectrum (solid curve) features several attenuation peaks corresponding to the excitation of plasmonic modes on the surface of metalized channels filled with aqueous analyte of refractive index 1.33. By changing the analyte refractive index (dotted curve), resonant attenuation peaks corresponding to the points of phase matching between the core-guided and plasmon modes shift. In the insets, energy flux of a core-guided-mode is presented at the two absorption peaks. Spectral position of the peak $\mathrm{I}$ is the most sensitive of the two peaks to changes in the analyte refractive index. This is because the corresponding core mode is coupled to a plasmon with a strong presence in the analyte region in contrast to the peak II at which plasmonic mode is predominantly extended into the cladding region.

leaky modes as, outside of a waveguide core, they do not exhibit a traditional evanescent decay into the cladding, but rather they radiate slowly (leak) into the cladding. Unlike in the case of common TIR waveguides, leaky modes in photonic crystal waveguides are confined by the bandgap of a photonic crystal reflector. As a consequence, the effective refractive index of the fundamental (lowest loss) leaky core mode can be designed to be arbitrarily smaller than that of a waveguide core material, thus enabling phase matching with a plasmon at any desired frequency. Moreover, the lowest loss 
leaky core mode typically exhibits a Gaussian-like intensity distribution in the waveguide core region, thus enabling convenient excitation by a Gaussian beam of an external light source. Using the fundamental (lowest loss) leaky mode for sensing gives the additional advantage of an effectively single mode propagation regime. In particular, when a set of modes is excited at a sensor input, higher-order leaky modes radiate out faster than a fundamental mode. Consequently, after a certain propagation distance, only the fundamental mode is left in the waveguide core. Finally, the effective angle of modal incidence onto a metal film, and hence sensitivity, can be varied at will by a proper selection of the waveguide core and reflector materials.

6.1. SPR Sensors Using Planar Photonic Bandgap Waveguides. To demonstrate the principles of operation of photonic bandgap waveguide-based SPR sensors we start by considering plasmon excitation by a Gaussian-like TMpolarized mode of a planar photonic crystal waveguide (see Figure 7(a)), in which light confinement in a lower refractive index core is achieved by a surrounding multilayer reflector. TM polarization of the electromagnetic field in a planar multilayer assumes a single magnetic field component $\left|H_{\|}\right|^{2}$ directed parallel to the plane of a multilayer, while the electric field component is confined to a plane perpendicular to the multilayer.

The photonic crystal waveguide under consideration consists of 27 alternating layers having refractive indices $n_{h}=2.0$, and $n_{l}=1.5$. The core layer is layer number 12; having refractive index $n_{c}=n_{l}$. Analyte (first cladding) is water $n_{a}=1.332$ bordering a $50 \mathrm{~nm}$ layer of gold. The substrate refractive index is 1.5 . Theory of planar photonic crystal waveguides with infinite reflectors where $n_{c}=n_{l}$ [64], predicts that, for a design wavelength $\lambda_{c}$, the effective refractive index $n_{\text {eff }}\left(\lambda_{c}\right)$ of the fundamental TE and TM coreguided-modes can be designed at will, as long as $0<n_{\text {eff }}<n_{l}$, by choosing the reflector layer thicknesses as

$$
d_{l, h}=\frac{\lambda_{c}}{4 \sqrt{n_{l, h}^{2}-n_{\mathrm{eff}}^{2}}},
$$

and by choosing the core layer thickness as $d_{c}=2 d_{l}$. Moreover, for this choice of $n_{c}$, the field distribution in the core is always Gaussian-like for TE-polarized modes, while for TM-polarized modes it is Gaussian-like as long as $n_{\text {eff }}^{2}>$ $\varepsilon_{l} \varepsilon_{h} /\left(\varepsilon_{l}+\varepsilon_{h}\right)$ [64]. By choosing the effective refractive index of a core mode to be that of a plasmon, a desired phase matching condition is achieved. For a waveguide with a finite reflector, the same design principle holds approximately. Thus, for an operating wavelength of $\lambda=640 \mathrm{~nm}$, considered in this example, phase matching is achieved when the photonic crystal waveguide is designed using $\lambda_{c}=635 \mathrm{~nm}$ and $\operatorname{Re}\left(n_{\text {eff }}\left(\lambda_{c}\right)\right)=1.46$ in (16). A reasonable approximation to the $n_{\text {eff }}\left(\lambda_{c}\right)$ is a value of the effective refractive index of a plasmonic wave propagating at a planar gold-analyte interface:

$$
n_{\mathrm{eff}}\left(\lambda_{c}\right)=\left(\frac{\varepsilon_{\mathrm{gold}}\left(\lambda_{c}\right) \cdot \varepsilon_{a}\left(\lambda_{c}\right)}{\varepsilon_{\mathrm{gold}}\left(\lambda_{c}\right)+\varepsilon_{a}\left(\lambda_{c}\right)}\right)^{1 / 2}
$$

where $\varepsilon_{a}$ is the dielectric constant of an analyte and $\varepsilon_{\text {gold }}$ is the dielectric constant of the gold layer approximated by the Drude model (15).

In Figure 7(b) we present band diagram of a planar photonic crystal waveguide-based SPR sensor. All the simulations are performed using standard transfer matrix theory on a complete system that includes both the waveguide and metal layer. Gray regions signify bulk states of a periodic reflector. The clear region is a TM bandgap where no extended into the reflector states are found. The thick solid line, which is almost parallel to the band gap edges, marked as "core mode" is a dispersion relation of a Gaussian-like leaky core mode with most of its modal energy concentrated in the low refractive index core. The dashed line marked as "plasmon" represents the dispersion relation of a plasmonic mode. Most of the plasmon energy is concentrated at the metal/analyte interface.

Near the phase matching point, fields of a core-guidedmode contain strong plasmonic contribution. As plasmon exhibits very high propagation loss, the loss of a core mode (upper plot in Figure 7(c)) will also exhibit a sharp increase near the phase matching point with a plasmon. An important aspect of the proposed setup is the freedom of adjusting the loss of a core mode. As leaky mode decays exponentially fast with respect to distance into the multilayer reflector, coupling strength between the plasmon and core modes can be controlled by changing the number of reflector layers situated between the waveguide core and a metal film. Ultimately, higher coupling strength leads to higher modal losses, hence, shorter sensor length. When the real part of the analyte refractive index is varied, the plasmon dispersion relation displaces accordingly, thus leading to a shift in the position of the phase matching point with a core-guided-mode. Consequently, in the vicinity of the phase matching point, transmission loss of a core-guided-mode varies strongly with changes in the analyte refractive index (see the upper part of Figure 7(c)).

We would like to point out that what is identified as a "core mode" in all the figures in this paper is in fact a waveguide supermode that includes both the core-guidedmode and plasmonic contribution. Since the plasmon mode is extremely lossy, only a small mixing of this mode with the core mode is necessary to achieve sensing. We found pertinent to also show on the graphs what a plasmonic mode looks like near the phase matching point, although this mode by itself is not used in our sensing arrangements. Depending on the designs, the plasmon contribution to the evanescent tail of a core mode is not always visible on the field distribution plots as only the real components of the modes are truly phase matched.

The simplest mode of operation of a waveguide-based SPR sensor is detection of small changes in the bulk refractive index of an analyte. Similarly to the case of hollow core PBG fiber-based sensors, there are two main modalities of SPR detection-amplitude-based and spectral-based. In both methodologies sensing is enabled through detection of changes in the location of a sharp plasmonic loss peak which spectral position is strongly dependent on the value of the ambient refractive index. In the amplitude-based 


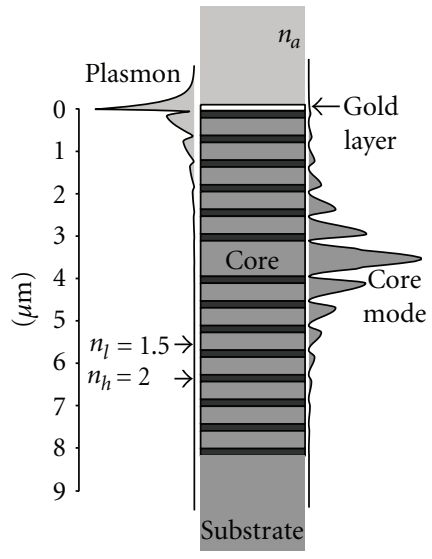

(a)

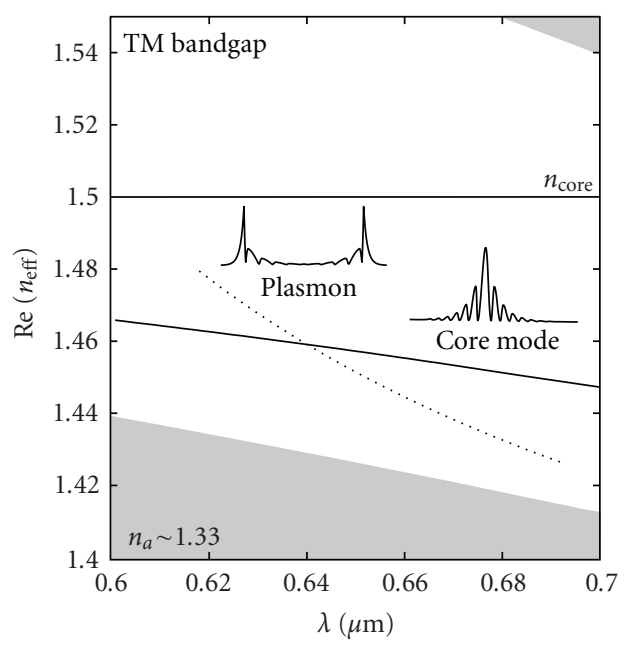

(b)
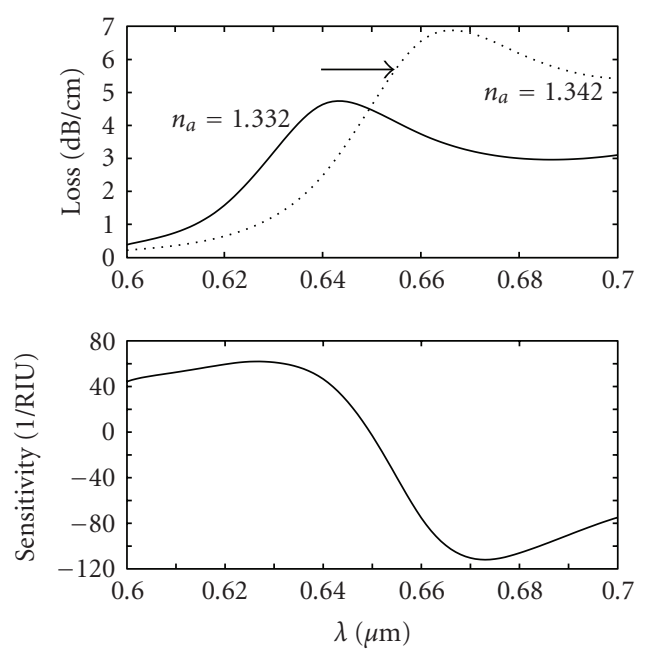

(c)

FIGURE 7: Planar photonic bandgap waveguide-based SPR sensor. (a) Schematic of a sensor. Low refractive index core is surrounded by the periodic photonic crystal reflector. Top side of the reflector is goldplated for plasmon excitation. Gold layer is bordered by aqueous analyte. $\left|H_{\|}\right|^{2}$ field distribution in the fundamental core mode is shown on the right, while field distribution in a plasmonic mode is show on the left of a sensor schematic. (b) Band diagram of sensor modes. Dispersion relation and field distribution of the fundamental core mode (thick solid curve), and plasmonic mode (dashed curve). TM bandgap of a periodic reflector is shown as a clear region, while gray regions correspond to the continuum of the bulk reflector states. By design, the effective refractive index of a core-guided-mode can be made significantly smaller than that of the waveguide core material. (c) Upper part: solid curve shows loss of a waveguide core mode near the phase matching point with a plasmon at which modal loss peaks. Dashed line shows shift of the modal loss curve when refractive index of the analyte is varied. Lower part: dependence of the sensor amplitude sensitivity on wavelength.

approach, all the amplitude measurements are performed at a single wavelength near the center of a plasmonic peak. The advantage of this method is its simplicity and low cost, as no spectral manipulation is required. The disadvantage is a smaller dynamic range and lower sensitivity when compared to the wavelength interrogation approach, in which the whole transmission spectra are taken and compared before and after the change in the analyte has occurred. We now use expression (5) to define sensor amplitude sensitivity with respect to changes in the real part of an analyte refractive index. In (5) the measurand $\delta$ is $\operatorname{Re}\left(\Delta n_{a}\right)$, and $\alpha(\delta, \lambda)$ is propagation loss of a core-guided-mode presented at the top of Figure 7(c). At the bottom of Figure 7(c) we present amplitude sensitivity of a PBG waveguide-based SPR sensor as a function of the wavelength of operation. Maximal sensitivity is achieved at $673 \mathrm{~nm}$ and is equal to $112 \mathrm{RIU}^{-1}$. It is typically a safe assumption that $1 \%$ change in the transmitted intensity can be detected reliably, which leads to a sensor resolution of $9 \cdot 10^{-5}$ RIU. In the wavelength interrogation mode, changes in the analyte refractive index are detected by measuring displacement of a plasmonic peak center wavelength $\lambda_{p}$ as a function of the value of an analyte refractive index. Sensor sensitivity is then defined by expression (7), where $\delta=\operatorname{Re}\left(\Delta n_{a}\right)$. We find that the spectral sensitivity of a PBG waveguide-based sensor is $2300 \mathrm{~nm}$. $\mathrm{RIU}^{-1}$. Assuming that a $0.1 \mathrm{~nm}$ change in the position of a resonance peak can be detected reliably, sensor resolution of $4.3 \cdot 10^{-5} \mathrm{RIU}$ is obtained.

Finally, sensor length is always in the range $L \sim 1 / \alpha(\delta, \lambda)$. In the vicinity of plasmonic peak shown in Figure 7 (c) typical sensor length is $L \sim 1 \mathrm{~cm}$. Detailed simulations also show that similarly to the case of resonant sensing using hollow core PBG fibers, sensitivity of a PBG waveguide-based plasmonic sensor is only weakly dependent on the sensor length. Particularly, by varying the number of reflector layers separating the waveguide core and gold layer one can vary the overall sensor length from sub-mm to several $\mathrm{cm}$ without changing significantly the sensor sensitivity.

In the rest of this section we present theoretical study of SPR sensor designs based on photonic bandgap fibers, rather than planar waveguides. Advantages of fiber based sensors over their planar counterparts include lower manufacturing cost, possibility of distributed sensing, and incorporation of microfluidics into the fiber structure directly at the fiber drawing step. In what follows we demonstrate an SPR sensor using solid core PBG Bragg fiber operating at $760 \mathrm{~nm}$, as well as an SPR sensor using honeycomb lattice photonic bandgap fiber operating at $1060 \mathrm{~nm}$.

6.2. SPR Sensors Using Photonic Bandgap Bragg Fibers. We start by describing solid core Bragg fiber-based SPR sensor for detection in aqueous analytes. In such a sensor a thin gold layer is deposited on the outer surface of a Bragg fiber in direct contact with an analyte. By tailoring the dispersion relation of the core-guided-mode of a Bragg fiber, phase matching with a plasmon can be obtained at any wavelength in the visible and near-IR. The mode of operation in such a sensor is a Gausian-like $H E_{11}$ core mode. Effective refractive index of such a mode is matched with that of a plasmon by the proper choice of the fiber core size. The choice of an $H E_{11}$ 
mode over the other modes is motivated by the ease of its excitation using common Gaussian laser sources.

As an example we consider solid core photonic crystal Bragg fibers made of two materials with refractive indices $n_{l}=1.42$ and $n_{l}=1.6$. Prototypes of such fibers have been recently fabricated in our group by using a poly(vinylene difloride) (PVDF)/polycarbonate (PC) material combination [65]. In such fibers, a solid core of refractive index $n_{l}$ is surrounded by $N$ alternating high and low refractive index reflector layers of thicknesses $d_{1}$, and $d_{h}$ (see Figure $8(\mathrm{a})$ ). In a manner similar to the planar multilayer waveguides, reflector layer thicknesses are given by the quarter wave condition (16), where $\lambda_{c}$ is an operating wavelength, and $n_{\text {eff }}\left(\lambda_{c}\right)$ is a desired effective refractive index of a core-guidedmode at that wavelength. Although such a choice of the reflector parameters guarantees bandgap guidance at $\lambda_{c}$ of a mode with effective refractive index $n_{\text {eff }}\left(\lambda_{c}\right)$, however, it does not guarantee existence of such a mode. One way of positioning a core mode dispersion relation inside of the reflector bandgap is by varying the fiber core diameter $d_{c}$. Namely, in the large core diameter Bragg fibers with $d_{c} \gg \lambda_{c}$, effective refractive index of the fundamental core mode is close to that of the core material. By decreasing the fiber core size, one can consistently reduce the core mode effective refractive index, and, eventually, position it in the middle of the reflector bandgap. Moreover, in the context of SPR sensing, $\lambda_{c}$ also corresponds to the wavelength of phase matching between plasmon and a core-guide mode. Therefore, a good approximation to $n_{\mathrm{eff}}\left(\lambda_{c}\right)$ of a core-guidedmode is that of the effective refractive index of a plasmonic wave propagating at a planar gold-analyte interface given by (17). With these choices of $n_{\text {eff }}\left(\lambda_{c}\right)$ and $d_{c}$ we are still left with one free parameter, which is the number of layers $N$ in the Bragg reflector. In metalized Bragg fibers, guided modes incur additional losses due to high absorption in the metal film. When operating within bandgap of a Bragg fiber reflector, the fields of leaky core modes decay exponentially fast into the reflector. Therefore, modal presence in the metal layer also decreases exponentially fast when increasing the number of reflector layers. Thus, the choice of the number of reflector layers primarily effects the core mode propagation loss, and, consequently, sensor length. As was mentioned earlier, PBG fiber-based sensor sensitivity is only weekly dependent on sensor length. Therefore, without the loss of sensitivity, one would choose a small enough number of reflector layers, so that the resultant fiber is short enough to prevent the necessity of coiling and simplify sensor handling.

In Figure 8 we present an example of a solid core PBG fiber-based SPR sensor. By choosing the fiber core size to be small, one can considerably reduce the effective refractive index of the core mode. This enables plasmonic excitation at longer wavelengths in the near-IR. In Figure 8(a) we show cross-section of a small-core Bragg fiber-based sensor, as well as energy flux distributions in the $H E_{11}$ core mode and plasmonic mode. Reflector layer thicknesses are chosen according to (16), where $\lambda_{c}=760 \mathrm{~nm}, n_{l}=1.42, n_{h}=$ 1.6 , and $n_{\mathrm{eff}}=1.39$, thus resulting in $n_{l}=654 \mathrm{~nm}$, $n_{l}=240 \mathrm{~nm}$. Fiber core diameter is $d_{c}=1.8 \mu \mathrm{m}$. The total number of layers is $N=12$. For the fundamental
Gaussian-like mode, the amount of energy in the core is $78 \%$. In Figure 8 (b) we present the band diagram of the modes of thus defined Bragg fiber sensor. Common TM, TE bandgap of a corresponding infinitely periodic Bragg reflector is presented as a clear region, while gray regions define a continuum of reflector bulk states. In a small core Bragg fiber, the effective refractive index of the core-guided $H E_{11}$ mode (thick solid line) can be considerably smaller than the refractive index of a core material (thin solid line). Dispersion relation of a plasmon mode is shown as thick dashed line. In this particular case, the dispersion relation of the core-guided-mode is shifted towards the lower edge of the reflector bandgap, therefore, the core mode (solid curve in Figure 8(a)) and plasmonic mode (dashed curve in Figure $8(\mathrm{a})$ ) penetrate significantly into the reflector. Phase matching between the core and plasmonic modes is achieved at $758 \mathrm{~nm}$. In the upper plot of Figure 8(c), propagation loss of the core-guided-mode is presented as a function of the wavelength. As seen from this plot, core mode loss peaks at the wavelength of phase matching with plasmon mode. In the lower plot of Figure 8(c), we present amplitude sensitivity (5) of a solid core Bragg fiber-based SPR sensor with respect to changes in the real part of the analyte refractive index. Maximal sensitivity is achieved at $788 \mathrm{~nm}$ and is equal to $293 \mathrm{RIU}^{-1}$. Assuming that a $1 \%$ change in the transmitted intensity can be detected reliably, this leads to a sensor resolution of $3.4 \cdot 10^{-5}$ RIU. Finally, we find that the corresponding spectral sensitivity (7) is $10^{4} \mathrm{~nm} \cdot \mathrm{RIU}^{-1}$. Assuming that a $0.1 \mathrm{~nm}$ change in the position of a resonance peak can be detected reliably, this leads to a sensor resolution of $9.8 \cdot 10^{-6}$ RIU. The sensor length in this case is in a $1 \mathrm{~cm}$ range.

6.3. SPR Sensors Using Photonic Bandgap Honeycomb Fibers. In the two previous subsections we have presented design strategies for the SPR sensors based on a PBG Bragg waveguide and a PBG Bragg fiber. In principle, any photonic bandgap fiber can be used in place of a Bragg fiber to develop such sensors. In this section we present an example of a SPR sensor based on a solid core honeycomb PBG fiber.

In Figure 9(a) schematic of a honeycomb photonic crystal fiber-based SPR sensor is presented. The design parameters are chosen as follows, the center-to-center distance between adjacent holes is $\Lambda=0.77 \mu \mathrm{m}$, the cladding hole diameter is $d=0.55 \Lambda$, the diameter of the hole in the core center is $d_{c}=0.35 \Lambda$. The fiber is made of silica glass with a refractive index of $n_{\text {glass }}=1.45$, the core and cladding holes are filled with air $n_{\text {air }}=1$, while the large semicircular channels are plated with a $40 \mathrm{~nm}$ thick layer of gold and filled with an aqueous analyte $n_{a}=1.32$. The central hole in the fiber core lowers its effective refractive index compared to that of a silica cladding. Under certain conditions, such a core can support a mode confined by the bandgap of the honeycomb reflector. The core-guided-mode in such a fiber is analogous to that of the small solid core Bragg fiber discussed earlier. Guided by the bandgap of the fiber reflector, the effective refractive index of the core mode can be made much lower than that of the silica material. 


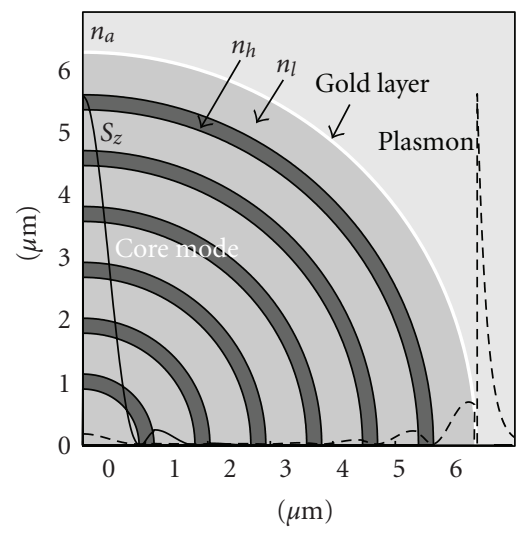

(a)

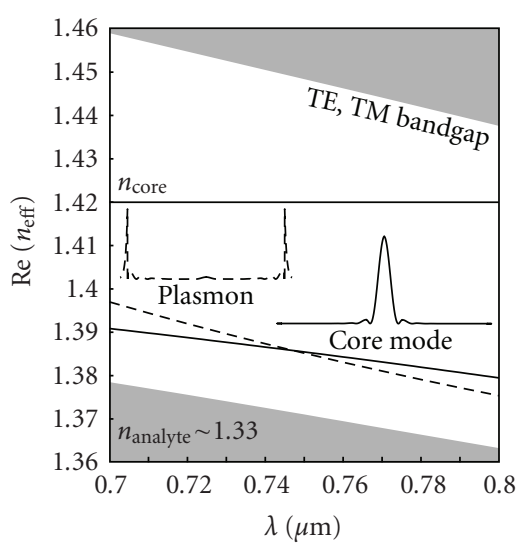

(b)
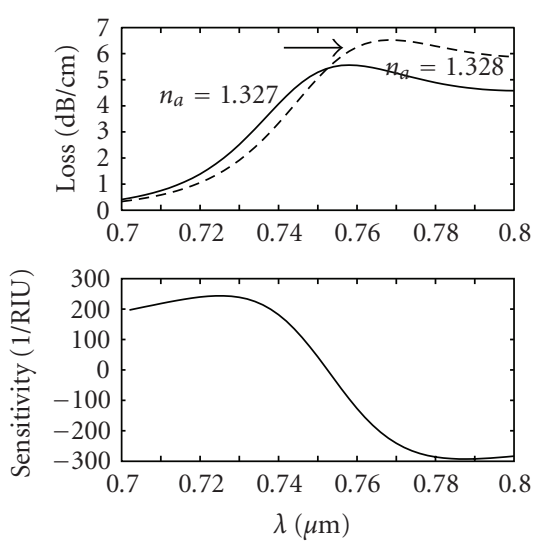

(c)

Figure 8: Small solid core PBG Bragg fiber-based SPR sensor. (a) Schematic of a sensor. Low refractive index core is surrounded by the concentric photonic crystal reflector. Reflector exterior is goldplated for plasmon excitation. Gold layer is bordered by aqueous analyte. Energy flux distribution across the fiber cross-section is shown as a solid curve for the fundamental core mode, and as a dashed curve for the plasmonic mode. (b) Band diagram of the sensor modes. Dispersion relation of the fundamental core mode (thick solid curve), and plasmonic mode (dashed curve). TE, TM bandgap of a periodic planar reflector is shown as a clear region, while gray regions correspond to the continuum of bulk reflector states. In a small core Bragg fiber, effective refractive index of the fundamental core mode can be much smaller than refractive index of the core material. (c) Upper plot: solid curve shows loss of the fundamental core mode near the phase matching point with plasmon. Modal loss reaches its maximum at the phase matching wavelength. Dashed line corresponds to the shifted modal loss curve when the analyte refractive index is varied. Lower plot: sensor amplitude sensitivity as a function of wavelength.

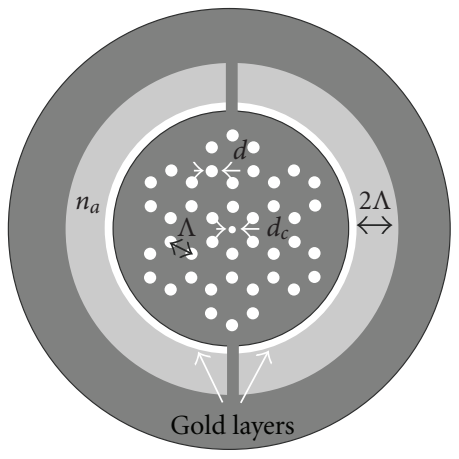

(a)

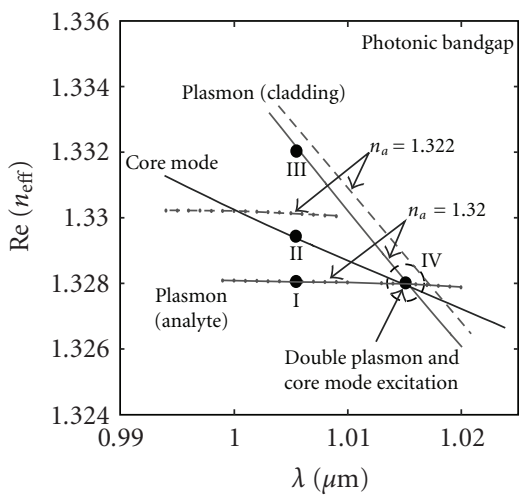

(b)
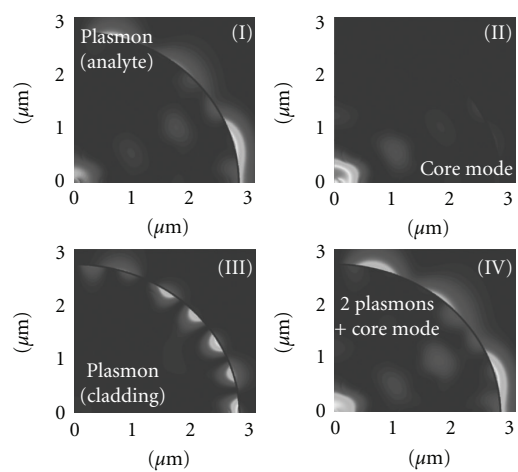

(c)

FIGURE 9: Solid core honeycomb photonic crystal fiber-based SPR sensor. (a) Schematic of a sensor. Solid fiber core having a small central hole is surrounded with a honeycomb photonic crystal reflector. Two large channels are integrated to implement analyte access to the fiber reflector region. The channels are goldplated for plasmon excitation. The gold layer is bordered by an aqueous analyte. (b) Band diagram of sensor modes. Dispersion relation of the fundamental core mode (thick solid curve), analyte bound plasmonic mode (dashed curve with circles), and cladding bound plasmonic mode (dashed curve). The bandgap of an infinitely periodic reflector is shown as a clear region. (c) The energy flux distributions across the fiber cross-section are shown for the fundamental core mode (II) as well as the analyte and cladding bound plasmon modes (I,III) outside of the phase matching region. The energy flux distribution is also shown for the fundamental core mode at the phase matching point (IV) showing strong mixing of the fundamental core mode with plasmonic modes.

Moreover, as in the case of photonic crystal Bragg fibers, radiation loss of a bandgap guided core mode can be reduced by adding more layers into the honeycomb reflector. The main reason why we chose a honeycomb structure of the fiber reflector is because it enables a very large photonic bandgap $[66,67]$, thus simplifying considerably phase matching of the core-guided and plasmonic modes.

Unlike planar metal/dielectric interface that supports a single plasmonic excitation, finite size, microstructured metal layer separating two dielectrics can support multiple plasmonic modes [51, 52]. Thus, when tracking losses of a core-guided fiber mode as a function of wavelength, one typically observes several plasmonic peaks corresponding to phase matching between the core mode and various plasmonic modes. Particularly, one of the plasmonic modes will have most of its energy concentrated in one of the neighboring dielectrics, while the other plasmonic excitation will have most of its energy concentrated in the other 


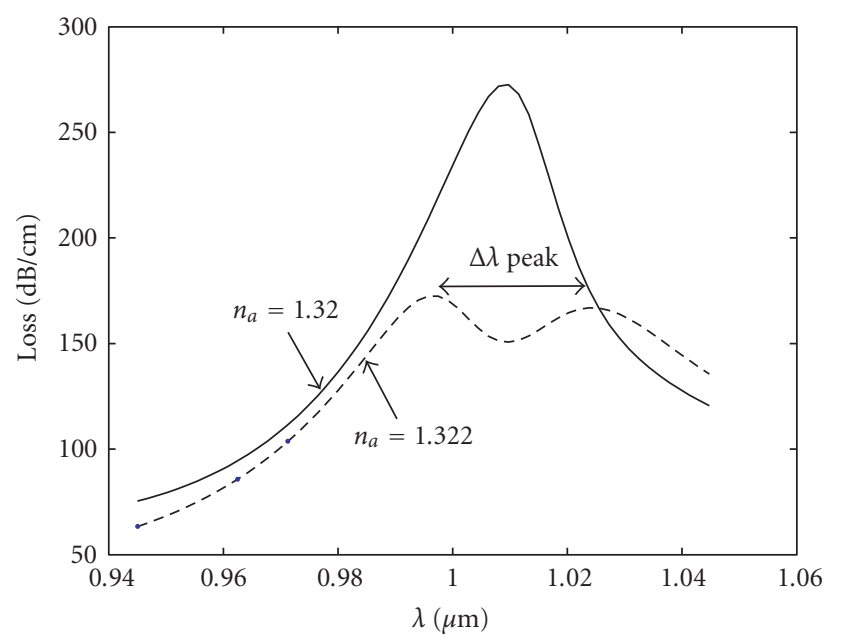

(a)

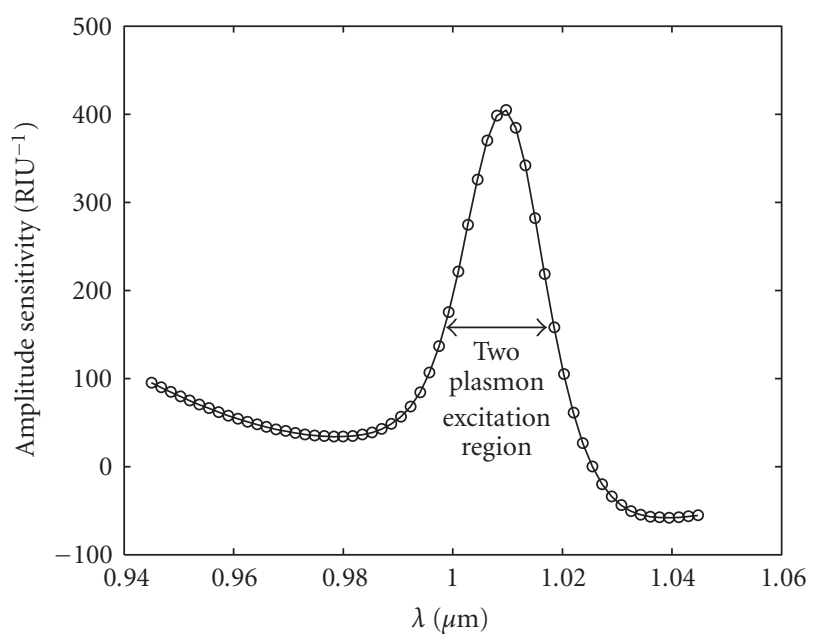

(b)

FIGURE 10: Sensitivity of the honeycomb photonic crystal fiber-based SPR sensor. (a) The solid curve shows loss of the fundamental core mode near the degenerate phase matching point with two plasmonic modes and $n_{a}=1.32$. Due to degeneracy, only one peak is distinguishable in the loss curve. Dashed line shows splitting of the degeneracy in plasmonic modes when the analyte refractive index is changed to $n_{a}=1.322$. (b) Dependence of the sensor amplitude sensitivity on wavelength.

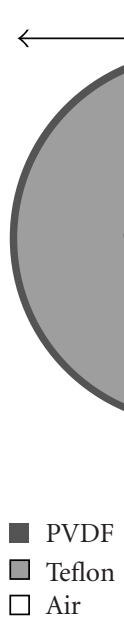

PVDF

Air
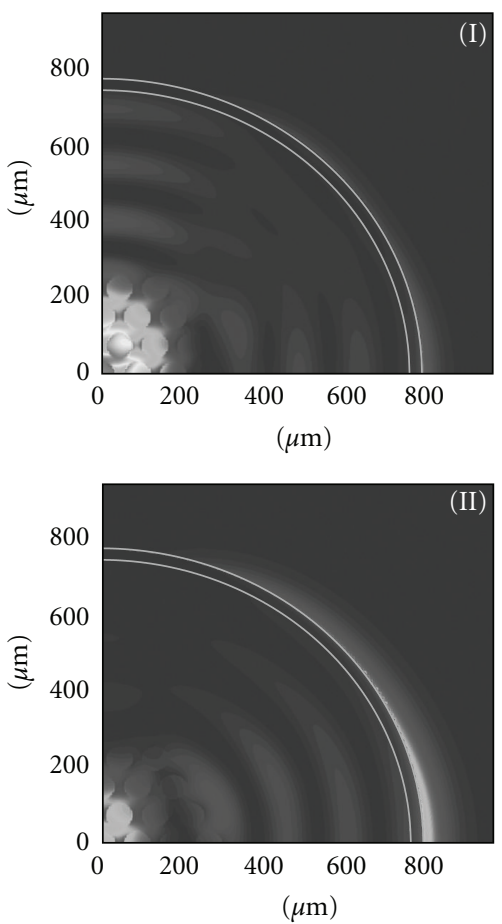

(b)

FIgURE 11: (a) Schematic of a porous THz fiber with a PVDF layer facing gaseous analyte. (b) Longitudinal energy flux distribution across the fiber crossection for the core-guided-mode far from the phase matching point with a Pseudoplasmon (I), and in the vicinity of a phase matching point with a Pseudoplasmon (II).

neighboring dielectric. In principle, simultaneous detection of changes in several plasmonic peaks can improve sensor sensitivity; additionally it gives a natural reference point in the measurements.

In the case of a honeycomb photonic crystal fiber-based sensor we design the fiber so that two plasmonic peaks are degenerate at $1009 \mathrm{~nm}$ with $n_{a}=1.32$. Figure $9(\mathrm{~b})$ shows the dispersion relations of the Gaussian-like core mode (thick solid line), analyte bound plasmonic mode (thin solid line with circles) and cladding bound plasmonic mode (thick solid line). These dispersion relationes are positioned well inside the bandgap of an infinite honeycomb reflector, 


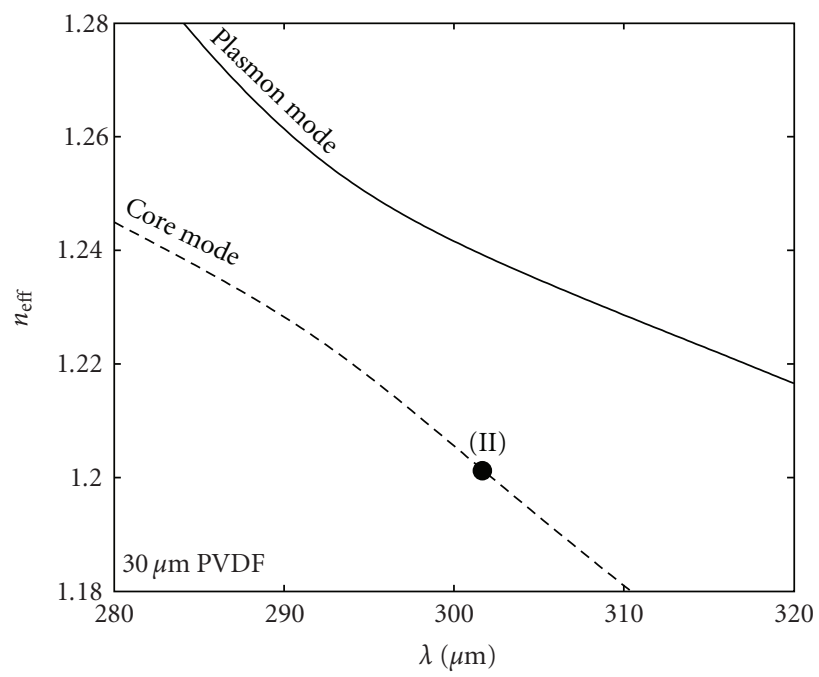

(a)

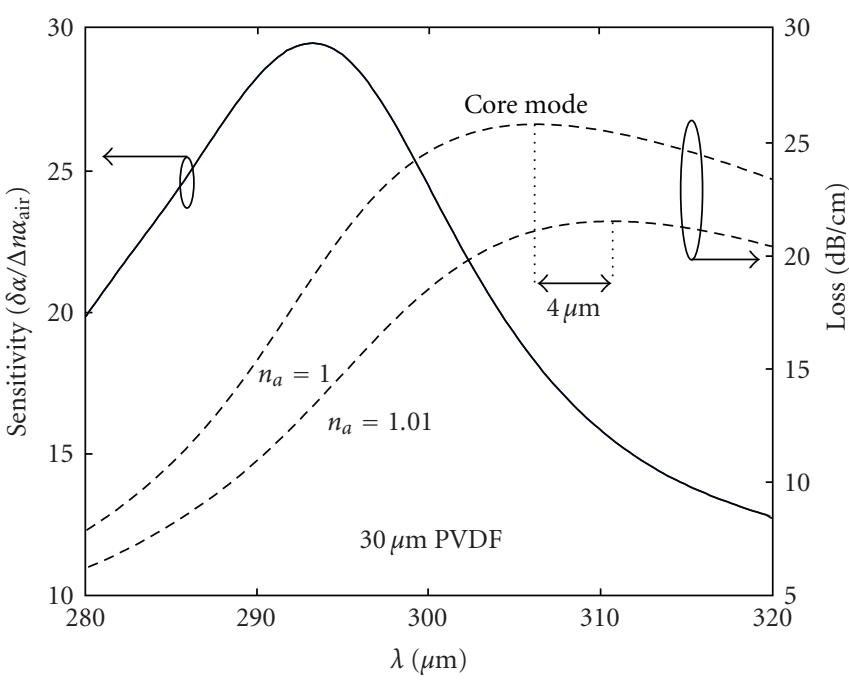

(b)

FIGURE 12: (a) Avoided crossing of the dispersion relations of the fundamental core mode (dashed curve) and a THz plasmon-like excitation (thin solid curve) in a porous fiber with a PVDF layer. (b) Losses of a core-guided-mode for two values of the gaseous analyte refractive index (dashed curves). A corresponding amplitude sensitivity curve for a sensor of the refractive index of a gaseous analyte is presented as a thick solid curve.

which can be confirmed by the plane wave method [67] Corresponding flux distributions of the core-guided and plasmonic modes are presented in Figure 9(c). The core mode loss shows a single plasmonic peak (solid curve in Figure 10(a). When the refractive index of the analyte is varied, this affects the two plasmonic dispersion relations differently. Particularly, the analyte bound plasmon mode is affected much strongly by the changes in the analyte refractive index than the cladding bound plasmonic mode. As a result, degeneracy is lifted, and two closely spaced plasmonic peaks appear in the core mode loss curve (dashed curve in Figure 10(a)). For example, a 0.002 change in the analyte refractive index splits a single plasmonic peak into two peaks separated by $27.5 \mathrm{~nm}$. This permits a novel spectral detection technique, where relative peak separation can be used to characterize changes in the real part of the analyte refractive index. By defining spectral sensitivity as

$$
S_{\lambda}=\lim _{\Delta n_{a} \rightarrow 0} \frac{\lambda_{\text {peak } 2}\left(n_{a}+\Delta n_{a}\right)-\lambda_{\text {peak } 1}\left(n_{a}+\Delta n_{a}\right)}{\Delta n_{a}},
$$

we find spectral sensitivity of $1.4 \cdot 10^{4} \mathrm{~nm} \cdot \mathrm{RIU}^{-1}$. It is typically a safe assumption that a $0.1 \mathrm{~nm}$ change in the position of a resonance peak can be detected reliably, which results in a sensor resolution of $7.2 \cdot 10^{-6} \mathrm{RIU}$, which is, to our knowledge, the highest reported spectral sensitivity of an aqueous fiber-based SPR sensor.

Finally, in Figure 10(b), we present the amplitude sensitivity of the proposed honeycomb photonic crystal fiberbased sensor as defined by (5). The maximal sensitivity is achieved at $1009 \mathrm{~nm}$ and equals to $400 \mathrm{RIU}^{-1}$. It is typically a safe assumption that a $1 \%$ change in the transmitted intensity can be detected reliably, which leads to a sensor resolution of $2.5 \cdot 10^{-5} \mathrm{RIU}$. It is interesting to note that for this sensor design, the sensitivity curve has a single maximum, unlike the sensitivity curves associated with the Bragg fiber designs reported in the preceding section. Sensor length in this case is in $1 \mathrm{~mm}$ range.

\section{Pseudoplasmon-Assisted Sensing Using Highly Porous Microstructured Fibers and Ferroelectric Layers in Terahertz Spectral Range}

In this final section we present an alternative approach to phase matching (at any desirable wavelength) a core-guided fiber mode with a plasmon. This approach is especially useful in the case of very low refractive index analytes, such as gases. As gaseous analytes have refractive index close to 1 , and as plasmon effective refractive index is generally close to that of an analyte, it becomes especially challenging to ensure that the effective refractive index of a core-guidedmode is low enough to match that of a plasmon. One way to lower the core mode effective refractive index is to work with subwavelength fibers or highly porous fibers that guide light while having a substantial fraction of their modal field located in the gaseous cladding. Unfortunately, subwavelength fibers in the visible and even in the midIR spectral range are difficult to fabricate and work with as their dimensions are smaller than a fraction of a $\mu \mathrm{m}$. However, in the terahertz $(\mathrm{THz})$ spectral range (also known as far-IR) that covers $30 \mu \mathrm{m}-3000 \mu \mathrm{m}$ wavelengths, the subwavelength fiber size is in tens or even hundreds of $\mu \mathrm{m}$, thus making such fibers easy to manufacture and handle [68].

An example of a subwavelength $\mathrm{THz}$ fiber that supports a mode with very low effective refractive index is presented in Figure 11(a). The fiber shown is a Teflon rod of $1480 \mu \mathrm{m}$ 
TABLE 1

\begin{tabular}{|c|c|c|c|c|c|}
\hline Sensor type & operation $\lambda$ & $\begin{array}{l}\text { Amplitude sensitivity } \\
S_{a}\left[\mathrm{RIU}^{-1}\right]\end{array}$ & $\begin{array}{l}\text { Sensor resolution (1\% } \\
\text { det. limit) } \operatorname{Re}\left(\Delta n_{a}\right)[\mathrm{RIU}]\end{array}$ & $\begin{array}{l}\text { Spectral } \\
\text { sensitivity } S_{\lambda}[\mathrm{nm} / \mathrm{RIU}]\end{array}$ & $\begin{array}{l}\text { Sensor resolution }(0.1 \mathrm{~nm} \\
\text { det. limit) } \operatorname{Re}\left(\Delta n_{a}\right)[\mathrm{RIU}]\end{array}$ \\
\hline $\begin{array}{l}\text { Analyte-filled } \\
\text { hollow core } \\
\text { Bragg fiber } \\
\text { (no metal). } \\
\text { Section } 3.2 \text {. }\end{array}$ & $\begin{array}{l}\text { anywhere in } \\
\text { the visible }\end{array}$ & 100 & $10^{-4}$ & 5300 & $2 \cdot 10^{-5}$ \\
\hline $\begin{array}{l}\text { Metalized } \\
\text { MOF. } \\
\text { Section } 5 .\end{array}$ & $\begin{array}{l}640 \mathrm{~nm} \\
\text { (Vis) }\end{array}$ & 180 & $6 \cdot 10^{-5}$ & & \\
\hline $\begin{array}{l}\text { Metalized } \\
\text { planar PBG } \\
\text { waveguide. } \\
\text { Section } 6.1\end{array}$ & $\begin{array}{l}673 \mathrm{~nm} \\
\text { (Vis) }\end{array}$ & 112 & $9 \cdot 10^{-5}$ & 2300 & $4.3 \cdot 10^{-5}$ \\
\hline $\begin{array}{l}\text { Metalized } \\
\text { solid core } \\
\text { Bragg fiber. } \\
\text { Section } 6.2 \\
\end{array}$ & $\begin{array}{l}788 \mathrm{~nm} \\
\text { (Vis-IR) }\end{array}$ & 293 & $3.4 \cdot 10^{-5}$ & 10000 & $9.8 \cdot 10^{-6}$ \\
\hline $\begin{array}{l}\text { Metalized } \\
\text { PBG photonic } \\
\text { crystal fiber. } \\
\text { Section } 6.3 \text {. }\end{array}$ & $\begin{array}{l}1009 \mathrm{~nm} \\
\text { (IR) }\end{array}$ & 400 & $2.5 \cdot 10^{-5}$ & 14000 & $7.2 \cdot 10^{-6}$ \\
\hline $\begin{array}{l}\text { PVDF- } \\
\text { activated } \\
\text { highly porous } \\
\text { subwave- } \\
\text { length fiber. } \\
\text { Section } 7 .\end{array}$ & $\begin{array}{l}292 \mu \mathrm{m} \\
(\mathrm{THz})\end{array}$ & 29 & $3.4 \cdot 10^{-4}$ & & \\
\hline
\end{tabular}

diameter having porous core in the form of 4 layers of hexagonally arranged subwavelength holes filled with air. The hole-to-hole distance (the pitch) is considered to be $\Lambda=$ $86 \mu \mathrm{m}$, while the hole diameter is $d=76 \mu \mathrm{m}$. The refractive indices of Teflon and air are 1.59 and 1.0 respectively. From outside, the teflon fiber is covered with a $30 \mu \mathrm{m}$ thick layer of a ferroelectric plastic (Polyvinylidene fluoride (PVDF)) facing air. PVDF is a ferroelectric semicrystalline polymer with a small absolute value of permittivity in the visible and near-IR regions. However, in the $\mathrm{THz}$ region the dielectric function of ferroelectric PVDF exhibits a resonance

$$
\varepsilon_{\mathrm{PVDF}}(\omega)=\varepsilon_{\mathrm{opt}}+\frac{\left(\varepsilon_{\mathrm{dc}}-\varepsilon_{\mathrm{opt}}\right) \omega_{\mathrm{TO}}^{2}}{\omega_{\mathrm{TO}}^{2}-\omega^{2}+i \gamma \omega},
$$

where, according to [69], $\varepsilon_{\mathrm{opt}}=2.0, \varepsilon_{\mathrm{dc}}=50.0, \omega_{\mathrm{TO}}=$ $0.3 \mathrm{THz}, \gamma=0.1 \mathrm{THz}$. In the wavelength range between $100 \mu \mathrm{m}(3 \mathrm{THz})$ and $700 \mu \mathrm{m}(\sim 0.43 \mathrm{THz})$ the real part of the refractive index of PVDF is less than one, while the imaginary part is positive and mostly larger than one. Therefore, the real part of the PVDF dielectric constant is negative and by analogy with the behavior of metals in the visible range, PVDF layer is expected to support a plasmon-like excitation, which we call a Pseudoplasmon.
As detailed in [68], introduction of subwavelength holes in the fiber core allows to lower dramatically the effective refractive index of the core-guided-mode. This, in turn, makes phase matching possible between the coreguided-mode and a $\mathrm{THz}$ Pseudoplasmon propagating at the PVDF/air interface [70]. Furthermore, outside of the phase matching point, most energy in the Gaussian-like core mode is guided in the subwavelength holes (as seen in the inset (I) of Figure 11(b)), thus resulting in greatly reduced modal absorption loss. In Figure 12 we present dispersion relation and losses of a core-guided and Pseudoplasmon modes. Particularly, Figure 12(a) presents the effective refractive index of the core-guided and Pseudoplasmon modes as a function of wavelength. Avoided mode crossing between the two modes around the wavelength of $300 \mu \mathrm{m}$ is clearly visible. In the inset (II) of Figure 11(b) we show field distribution in a hybrid mode in the vicinity of a phase matching point (point (II) in Figure 12(a)). From the plot, contributions from both the core-guided-mode and a Pseudoplasmon are clearly visible. In Figure 12(b), losses of a core-guidedmode near the point of avoided crossing are shown for two values of the analyte refractive index $n_{a}=1.0$ and $n_{a}=1.01$. Losses of a core-guided-mode peak $(\sim 25 \mathrm{~dB} / \mathrm{cm})$ at the point of phase matching with a Pseudoplasmon, while being much lower $(<10 \mathrm{~dB} / \mathrm{cm})$ outside of the phase matching region. In these simulations Teflon material is considered lossless compared to PVDF, which is justified as 
the bulk absorption loss of Teflon is reported to be as low as $\sim 1.3 \mathrm{~dB} / \mathrm{cm}$.

Finally, in Figure 12(b) we present amplitude sensitivity of the porous-core fiber-based sensor to changes in the real part of the analyte refractive index (see definition (5)). The maximal sensitivity is achieved at $292 \mu \mathrm{m}$ and equals to $29 \mathrm{RIU}^{-1}$. Assuming that $1 \%$ change in the transmitted intensity can be reliably detected, sensor resolution of 3.4 . $10^{-4}$ RIU is predicted. Sensor length in this case is in $1 \mathrm{~cm}$ range.

\section{Discussions}

First, we would like to comment on the limitations of theoretical estimates of the sensor detection limits. Throughout the paper we assumed, for example, that the spectral detection method allows detection of $0.1 \mathrm{~nm}$ changes in the position of resonant absorption peaks. If a resonant peak preserves its shape when the refractive index of analyte changes, then sensor resolution is only determined by the resolution of a spectrometer. This is indeed the case in our theoretical simulations. However, if noise is present, such as thermal noise, nonhomogeneity in the analyte refractive index etc., then the peak shape at maxima could change unpredictably between measurements, thus limiting the sensor resolution. The same argument applies to amplitude sensing where measurements are performed at the wavelength of strongest change in the waveguide loss. In our theoretical estimates we used spectral resolution of $0.1 \mathrm{~nm}$ and an amplitude resolution of $1 \%$ reflecting the available experimental body of work in resonant sensors. We note that these optical detection limits were achieved in real sensor systems of moderate complexity and cost.

Another important question regarding proposed sensors is about complexity of their practical implementation. As most of the presented designs use nanothick metallic layers deposited on the inner or outer surfaces of the fiber microstructure the question is about experimental technique that can allow such metallization. After the first paper on resonant MOF-based plasmonic sensors [53] there have been several reports of fabrication of the metal-coated and metal-filled photonic crystal fibers. Particularly, in [71] the authors reported deposition of thin metallic layers of silver on the inner walls of micron-sized holes of a MOF using chemical deposition via precipitation from a reduction reaction. In [72] the same reduction reaction was used to create silver nanoparticles on the microstructure walls. In [73] the authors used high pressure chemical deposition process to deposit nanoparticles and nanothick layers of silver onto the inner walls of the microstructured fibers. In [74] the authors have codrawn a copper rod sealed in a silica tube, then inserted a thus drawn copper/silica wire into a silica fiber preform and redraw it again to get a MOF with selectively metalized holes. Finally, the first demonstration of a photonic bandgap fiber-based plasmonic sensor was presented in [75] where the authors deposited gold layer onto the outer surface of a tapered Bragg fiber. A rapid progress in the fabrication of metalized MOFs and POFs makes us to believe that various sensors presented in this work indeed have a strong practical potential.

\section{Conclusions}

In conclusion, we have reviewed microstructured optical fiber and photonic bandgap (PBG) fiber-based resonant optical sensors of changes in the real part of the analyte refractive index. Particularly, we have considered two sensors types. One sensor type employed hollow core photonic bandgap fibers where core-guided-mode is strongly confined in the analyte-filled core. Another sensor type employed metalized photonic bandgap waveguides and fibers, where core-guided-mode was phase matched with a plasmon propagating at the metalized fiber/analyte interface. All the sensor types described in the paper showed strong resonant dependence of the fiber absorption on the value of the analyte refractive index, leading to the $10^{-6}-10^{-4}$ RIU resolution in the real part of the aqueous and gaseous analyte refractive index (assuming amplitude or spectral interrogation techniques). In both sensor types the length is designable and can range from submillimeters to several meters. Surprisingly, we have discovered that the maximal sensor sensitivity is largely independent of the sensor length. Finally, operational regions of the resonant sensors reviewed in this work spanned a wide range of wavelengths from the visible to terahertz.

To summarize the results of this paper, we also present a table of performance parameters of all the resonant sensor designs considered in this paper. In all but the last designs we assume an aqueous analyte of $n_{a} \approx 1.32$, while in the case of $\mathrm{THz}$ sensor we assume a gaseous analyte $n_{a} \approx 1$.

\section{References}

[1] A. MacLean, C. Moran, W. Johnstone, B. Culshaw, D. Marsh, and P. Parker, "Detection of hydrocarbon fuel spills using a distributed fibre optic sensor," Sensors and Actuators A, vol. 109, no. 1-2, pp. 60-67, 2003.

[2] N. Kim, I.-S. Park, and W.-Y. Kim, "Salmonella detection with a direct-binding optical grating coupler immunosensor," Sensors and Actuators B, vol. 121, no. 2, pp. 606-615, 2007.

[3] S. Balasubramanian, I. B. Sorokulova, V. J. Vodyanoy, and A. L. Simonian, "Lytic phage as a specific and selective probe for detection of Staphylococcus aureus - a surface plasmon resonance spectroscopic study," Biosensors and Bioelectronics, vol. 22, no. 6, pp. 948-955, 2007.

[4] H. J. Watts, C. R. Lowe, and D. V. Pollard-Knight, "Optical biosensor for monitoring microbial cells," Analytical Chemistry, vol. 66, no. 15, pp. 2465-2470, 1994.

[5] D. R. DeMarco and D. V. Lim, "Direct detection of Escherichia coli 0157:H7 in unpasteurized apple juice with an evanescent wave biosensor," Journal of Rapid Methods and Automation in Microbiology, vol. 9, no. 4, pp. 241-257, 2001.

[6] J. R. E. Shepard, Y. Danin-Poleg, Y. Kashi, and D. R. Walt, "Array-based binary analysis for bacterial typing," Analytical Chemistry, vol. 77, no. 1, pp. 319-326, 2005.

[7] M. Zourob, S. Mohr, B. J. T. Brown, P. R. Fielden, M. B. McDonnell, and N. J. Goddard, "An integrated metal clad 
leaky waveguide sensor for detection of bacteria," Analytical Chemistry, vol. 77, no. 1, pp. 232-242, 2005.

[8] J. B. Jensen, P. E. Hoiby, G. Emiliyanov, O. Bang, L. H. Pedersen, and A. Bjarklev, "Selective detection of antibodies in microstructured polymer optical fibers," Optics Express, vol. 13, no. 15, pp. 5883-5889, 2005.

[9] J. M. Fini, "Microstructure fibres for optical sensing in gases and liquids," Measurement Science and Technology, vol. 15, no. 6, pp. 1120-1128, 2004.

[10] S. O. Konorov, A. M. Zheltikov, and M. Scalora, "Photoniccrystal fiber as a multifunctional optical sensor and sample collector," Optics Express, vol. 13, no. 9, pp. 3454-3459, 2005.

[11] E. Pone, C. Dubois, N. Guo, et al., "Drawing of the hollow allpolymer Bragg fibers," Optics Express, vol. 14, no. 13, p. 5838, 2006.

[12] B. Temelkuran, S. D. Hart, G. Benoit, J. D. Joannopoulos, and Y. Fink, "Wavelength-scalable hollow optical fibres with large photonic bandgaps for $\mathrm{CO}_{2}$ laser transmission," Nature, vol. 420, no. 6916, pp. 650-653, 2002.

[13] J. C. Knight, T. A. Birks, P. St. J. Russell, and J. G. Rarity, "Bragg scattering from an obliquely illuminated photonic crystal fiber," Applied Optics, vol. 37, no. 3, pp. 449-452, 1998.

[14] P. St. J. Russell, "Photonic-crystal fibers," Journal of Lightwave Technology, vol. 24, no. 12, pp. 4729-4749, 2006.

[15] C. M. Smith, N. Venkataraman, M. T. Gallagher, et al., "Lowloss hollow-core silica/air photonic bandgap fibre," Nature, vol. 424, no. 6949, pp. 657-659, 2003.

[16] G. Vienne, Y. Xu, C. Jakobsen, et al., "Ultra-large bandwidth hollow-core guiding in all-silica Bragg fibers with nanosupports," Optics Express, vol. 12, no. 15, pp. 3500-3508, 2004.

[17] A. Argyros, M. A. van Eijkelenborg, M. C. J. Large, and I. M. Bassett, "Hollow-core microstructured polymer optical fiber," Optics Letters, vol. 31, no. 2, pp. 172-174, 2006.

[18] J. A. Harrington, "Review of IR transmitting, hollow waveguides," Fiber and Integrated Optics, vol. 19, no. 3, pp. 211-227, 2000.

[19] Y. W. Shi, K. Ito, Y. Matsuura, and M. Miyagi, "Multiwavelength laser light transmission of hollow optical fiber from the visible to the mid-infrared," Optics Letters, vol. 30, no. 21, pp. 2867-2869, 2005.

[20] Y. L. Hoo, W. Jin, H. L. Ho, J. Ju, and D. N. Wang, "Gas diffusion measurement using hollow-core photonic bandgap fiber," Sensors and Actuators B, vol. 105, no. 2, pp. 183-186, 2005.

[21] C. Charlton, B. Temelkuran, G. Dellemann, and B. Mizaikoff, "Midinfrared sensors meet nanotechnology: trace gas sensing with quantum cascade lasers inside photonic band-gap hollow waveguides," Applied Physics Letters, vol. 86, no. 19, Article ID 194102, 3 pages, 2005.

[22] F. M. Cox, A. Argyros, and M. C. J. Large, "Liquid-filled hollow core microstructured polymer optical fiber," Optics Express, vol. 14, no. 9, pp. 4135-4140, 2006.

[23] S. Smolka, M. Barth, and O. Benson, "Highly efficient fluorescence sensing with hollow core photonic crystal fibers," Optics Express, vol. 15, no. 20, pp. 12783-12791, 2007.

[24] A. W. Snyder and J. Love, Optical Waveguide Theory, Springer, London, UK, 2nd edition, 2008.

[25] S. G. Johnson, M. Ibanescu, M. Skorobogatiy, et al., "Lowloss asymptotically single-mode propagation in large-core omniguide fibers," Optics Express, vol. 9, no. 13, pp. 748-779, 2001.

[26] M. Skorobogatiy, "Efficient antiguiding of TE and TM polarizations in low-index core waveguides without the need for an omnidirectional reflector," Optics Letters, vol. 30, no. 22, pp. 2991-2993, 2005.

[27] V. M. Agranovich and D. L. Mills, Surface Polaritons: Electromagnetic Waves at Surfaces and Interfaces, North-Holland, Amsterdam, The Netherlands, 1982.

[28] E. Kretschmann and H. Raether, "Radiative decay of non radiative surface plasmons excited by light," Naturforschung A, vol. 23, p. 2135, 1968.

[29] B. Liedberg, C. Nylander, and I. Lundström, "Surface plasmon resonance for gas detection and biosensing," Sensors and Actuators, vol. 4, pp. 299-304, 1983.

[30] J. Melendez, R. Carr, D. U. Bartholomew, et al., "A commercial solution for surface plasmon sensing," Sensors and Actuators B, vol. 35, no. 1-3, pp. 212-216, 1996.

[31] L.-M. Zhang and D. Uttamchandani, "Optical chemical sensing employing surface plasmon resonance," Electronics Letters, vol. 24, no. 23, pp. 1469-1470, 1988.

[32] A. V. Kabashin and P. I. Nikitin, "Surface plasmon resonance interferometer for bio- and chemical-sensors," Optics Communications, vol. 150, no. 1-6, pp. 5-8, 1998.

[33] A. N. Grigorenko, P. I. Nikitin, and A. V. Kabashin, "Phase jumps and interferometric surface plasmon resonance imaging," Applied Physics Letters, vol. 75, no. 25, pp. 3917-3919, 1999.

[34] M. Manuel, B. Vidal, R. López, et al., "Determination of probable alcohol yield in musts by means of an SPR optical sensor," Sensors and Actuators B, vol. 11, no. 1-3, pp. 455-459, 1993.

[35] R. Alonso, J. Subias, J. Pelayo, F. Villuendas, and J. Tornos, "Single-mode, optical-fiber sensors and tunable wavelength filters based on the resonant excitation of metal-clad modes," Applied Optics, vol. 33, no. 22, pp. 5197-5201, 1994.

[36] J. Homola, "Optical fiber sensor based on surface plasmon excitation," Sensors and Actuators B, vol. 29, no. 1-3, pp. 401405, 1995.

[37] A. J. C. Tubb, F. P. Payne, R. B. Millington, and C. R. Lowe, "Single-mode optical fibre surface plasma wave chemical sensor," Sensors and Actuators B, vol. 41, no. 1-3, pp. 71-79, 1997.

[38] J. Homola, J. Čtyrocký, M. Skalský, J. Hradilová, and P. Kolárová, "A surface plasmon resonance based integrated optical sensor," Sensors and Actuators B, vol. 39, no. 1-3, pp. 286-290, 1997.

[39] A. Díez, M. V. Andrés, and J. L. Cruz, "In-line fiber-optic sensors based on the excitation of surface plasma modes in metal-coated tapered fibers," Sensors and Actuators B, vol. 73, no. 2-3, pp. 95-99, 2001.

[40] M. Piliarik, J. Homola, Z. Maníková, and J. Čtyroký, "Surface plasmon resonance sensor based on a single-mode polarization-maintaining optical fiber," Sensors and Actuators B, vol. 90, no. 1-3, pp. 236-242, 2003.

[41] D. Monzón-Hernández, J. Villatoro, D. Talavera, and D. LunaMoreno, "Optical-fiber surface-plasmon resonance sensor with multiple resonance peaks," Applied Optics, vol. 43, no. 6, pp. 1216-1220, 2004.

[42] D. Monzón-Hernández and J. Villatoro, "High-resolution refractive index sensing by means of a multiple-peak surface plasmon resonance optical fiber sensor," Sensors and Actuators $B$, vol. 115, no. 1, pp. 227-231, 2006.

[43] H. Suzuki, M. Sugimoto, Y. Matsui, and J. Kondoh, "Fundamental characteristics of a dual-colour fibre optic SPR sensor," Measurement Science and Technology, vol. 17, no. 6, pp. 15471552, 2006. 
[44] S. J. Al-Bader and M. Imtaar, "Optical fiber hybrid-surface plasmon polaritons," Journal of the Optical Society of America $B$, vol. 10, p. 83, 1993.

[45] R. C. Jorgenson and S. S. Yee, "A fiber-optic chemical sensor based on surface plasmon resonance," Sensors and Actuators B, vol. 12, no. 3, pp. 213-220, 1993.

[46] A. Trouillet, C. Ronot-Trioli, C. Veillas, and H. Gagnaire, "Chemical sensing by surface plasmon resonance in a multimode optical fibre," Pure and Applied Optics, vol. 5, no. 2, pp. 227-237, 1996.

[47] J. Čtyrocký, J. Homola, P. V. Lambeck, et al., “Theory and modelling of optical waveguide sensors utilising surface plasmon resonance," Sensors and Actuators B, vol. 54, no. 1, pp. 66-73, 1999.

[48] M. Weisser, B. Menges, and S. Mittler-Neher, "Refractive index and thickness determination of monolayers by multi mode waveguide coupled surface plasmons," Sensors and Actuators B, vol. 56, no. 3, pp. 189-197, 1999.

[49] B. D. Gupta and A. K. Sharma, "Sensitivity evaluation of a multi-layered surface plasmon resonance-based fiber optic sensor: a theoretical study," Sensors and Actuators B, vol. 107, no. 1, pp. 40-46, 2005.

[50] B. T. Kuhlmey, K. Pathmanandavel, and R. C. McPhedran, "Multipole analysis of photonic crystal fibers with coated inclusions," Optics Express, vol. 14, no. 22, pp. 10851-10864, 2006.

[51] A. Hassani and M. Skorobogatiy, "Design of the microstructured optical fiber-based surface plasmon resonance sensors with enhanced microfluidics," Optics Express, vol. 14, no. 24, pp. 11616-11621, 2006.

[52] B. Gauvreau, A. Hassani, M. F. Fehri, A. Kabashin, and M. Skorobogatiy, "Photonic bandgap fiber-based surface plasmon resonance sensors," Optics Express, vol. 15, no. 18, pp. 1141311426, 2007.

[53] M. Skorobogatiy and A. V. Kabashin, "Photon crystal waveguide-based surface plasmon resonance biosensor," Applied Physics Letters, vol. 89, no. 14, Article ID 211641, 2006.

[54] C. R. Lavers and J. S. Wilkinson, "A waveguide-coupled surface-plasmon sensor for an aqueous environment," Sensors and Actuators B, vol. 22, no. 1, pp. 75-81, 1994.

[55] R. D. Harris and J. S. Wilkinson, "Waveguide surface plasmon resonance sensors," Sensors and Actuators B, vol. 29, no. 1-3, pp. 261-267, 1995.

[56] M. N. Weiss, R. Srivastava, and H. Groger, "Experimental investigation of a surface plasmon-based integrated-optic humidity sensor," Electronics Letters, vol. 32, no. 9, pp. 842843,1996

[57] J. Dostáleka, J. Tyrokýa, J. Homola, et al., "Surface plasmon resonance biosensor based on integrated optical waveguide," Sensors and Actuators B, vol. 76, no. 1-3, pp. 8-12, 2001.

[58] A. K. Sheridan, R. D. Harris, P. N. Bartlett, and J. S. Wilkinson, "Phase interrogation of an integrated optical SPR sensor," Sensors and Actuators B, vol. 97, no. 1, pp. 114-121, 2004.

[59] A. Hassani and M. Skorobogatiy, "Design criteria for microstructured-optical-fiber-based surface-plasmonresonance sensors," Journal of the Optical Society of America B, vol. 24, no. 6, pp. 1423-1429, 2007.

[60] C. M. B. Cordeiro, M. A. R. Franco, G. Chesini, et al., "Microstructured-core optical fibre for evanescent sensing applications," Optics Express, vol. 14, no. 26, pp. 13056-13066, 2006.

[61] I. H. Malitson, "Interspecimen comparison of the refractive index of fused silica," Journal of the Optical Society of America, vol. 55, pp. 1205-1209, 1965.
[62] S. Patskovsky, A. V. Kabashin, and M. Meunier, "Near-infrared surface plasmon resonance sensing on a Si platform with nanoparticle-based signal enhancement," Optical Materials, vol. 27, no. 5, pp. 1093-1096, 2005.

[63] L. O. Cinteza, T. Y. Ohulchanskyy, Y. Sahoo, E. J. Bergey, R. K. Pandey, and P. N. Prasad, "Diacyllipid micelle-based nanocarrier for magnetically guided delivery of drugs in photodynamic therapy," Molecular Pharmaceutics, vol. 3, no. 4, pp. 415-423, 2006.

[64] M. Skorobogatiy, "Efficient antiguiding of TE and TM polarizations in low-index core waveguides without the need for an omnidirectional reflector," Optics Letters, vol. 30, no. 22, pp. 2991-2993, 2005.

[65] Y. Gao, N. Guo, B. Gauvreau, et al., "Consecutive solvent evaporation and co-rolling techniques for polymer multilayer hollow fiber preform fabrication," Journal of Materials Research, vol. 21, no. 9, pp. 2246-2254, 2006.

[66] T. Murao, K. Saitoh, and M. Koshiba, "Design of air-guiding modified honeycomb photonic band-gap fibers for effectively single-mode operation," Optics Express, vol. 14, no. 6, pp. 2404-2412, 2006.

[67] S. E. Barkou, J. Broeng, and A. Bjarklev, "Silica-air photonic crystal fiber design that permits waveguiding by a true photonic bandgap effect," Optics Letters, vol. 24, no. 1, pp. 46$48,1999$.

[68] T. Hidaka, H. Minamide, H. Ito, J.-I. Nishizawa, K. Tamura, and S. Ichikawa, "Ferroelectric PVDF cladding terahertz waveguide," Journal of Lightwave Technology, vol. 23, no. 8, pp. 2469-2473, 2005.

[69] A. Hassani, A. Dupuis, and M. Skorobogatiy, "Low loss porous terahertz fibers containing multiple subwavelength holes," Applied Physics Letters, vol. 92, no. 7, Article ID 071101, 2008.

[70] A. Hassani, A. Dupuis, and M. Skorobogatiy, "Porous polymer fibers for low-loss Terahertz guiding," Optics Express, vol. 16, no. 9, pp. 6340-6351, 2008.

[71] X. Zhang, R. Wang, F. M. Cox, B. T. Kuhlmey, and M. C. J. Large, "Selective coating of holes in microstructured optical fiber and its application to in-fiber absorptive polarizers," Optics Express, vol. 15, no. 24, pp. 16270-16278, 2007.

[72] X. Yang and L. Wang, "Silver nanocrystals modified microstructured polymer optical fibres for chemical and optical sensing," Optics Communications, vol. 280, no. 2, pp. 368-373, 2007.

[73] A. C. Peacock, A. Amezcua-Correa, J. Yang, P. J. A. Sazio, and S. M. Howdle, "Highly efficient surface enhanced Raman scattering using microstructured optical fibers with enhanced plasmonic interactions," Applied Physics Letters, vol. 92, no. 14, Article ID 141113, 2008.

[74] J. Hou, D. Bird, A. George, S. Maier, B. T. Kuhlmey, and J. C. Knight, "Metallic mode confinement in microstructured fibres," Optics Express, vol. 16, no. 9, pp. 5983-5990, 2008.

[75] L. Ma, T. Katagiri, and Y. Matsuura, "Surface-plasmon resonance sensor using silica-core Bragg fiber," Optics Letters, vol. 34, no. 7, pp. 1069-1071, 2009. 

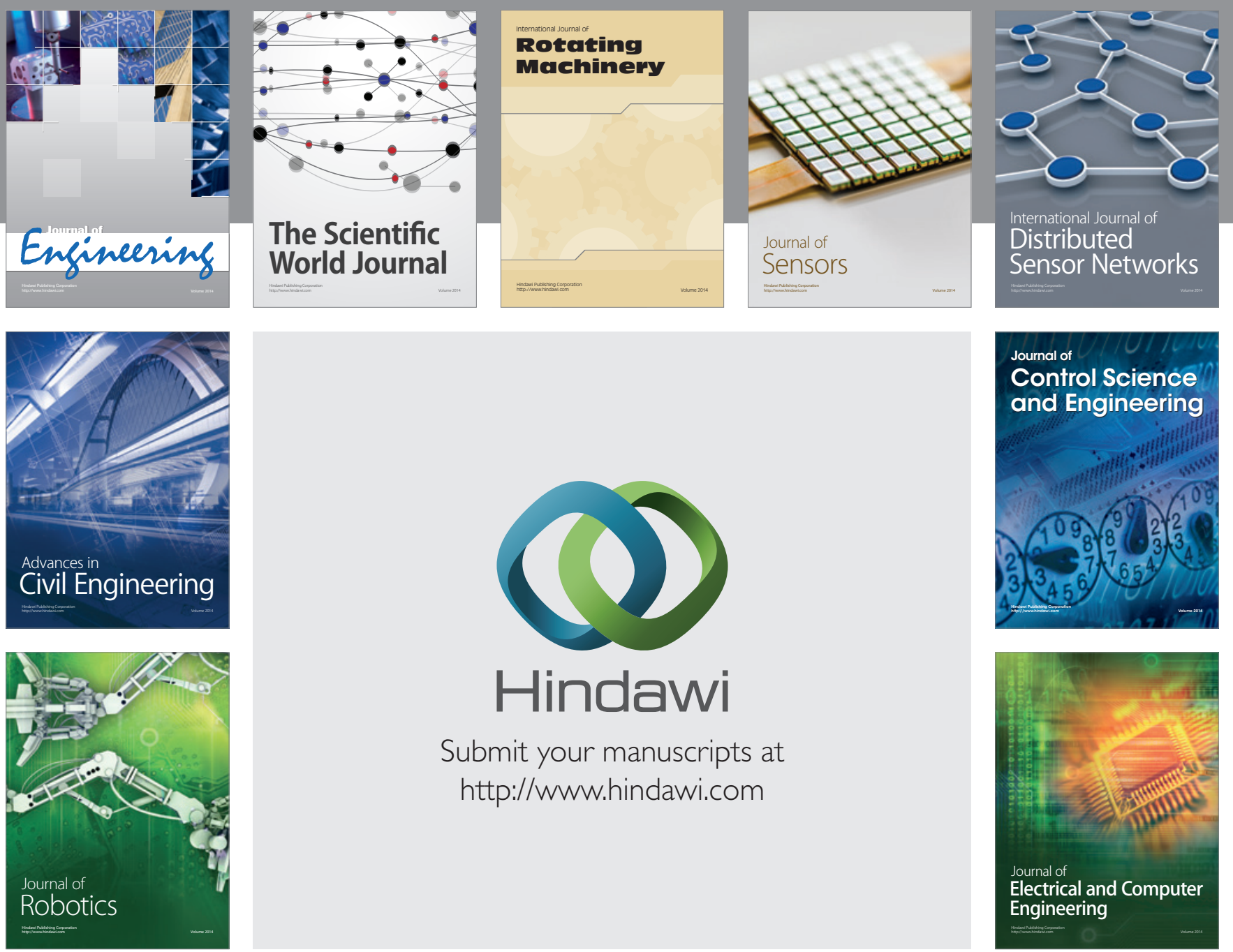

Submit your manuscripts at

http://www.hindawi.com
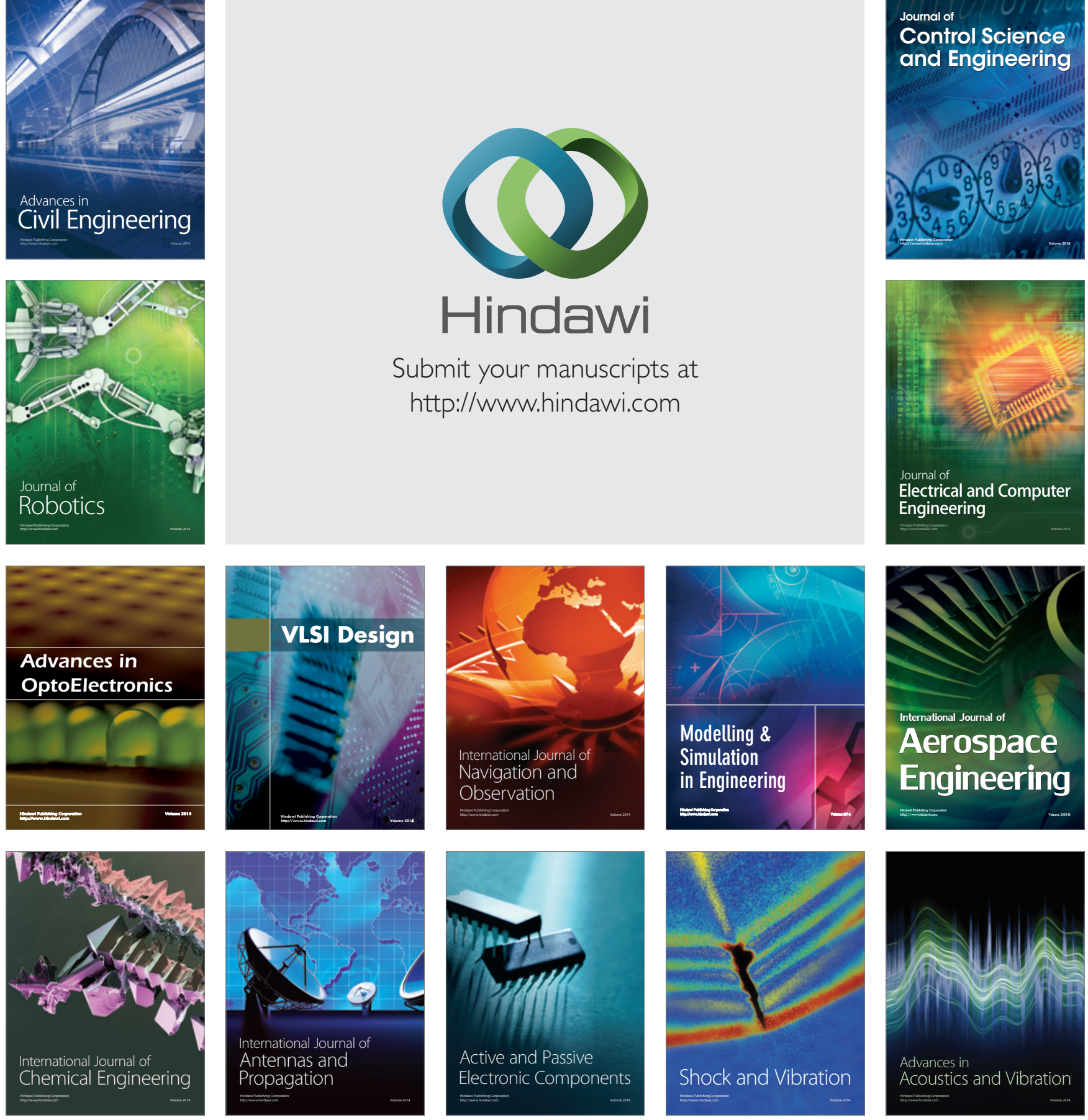